\title{
HIERARCHICAL CONFIGURATIONS FOR CROSS-CORRELATION INTERFEROMETERS WITH MANY ELEMENTS
}

\author{
ERIC KETO \\ Harvard-Smithsonian Center for Astrophysics \\ 60 Garden St, Cambridge, MA 02138 \\ keto@cfa.harvard.edu \\ Received 2012 May 29; Revised 2012 August 30; Accepted 2012 September 3; Published 2012 October 19

\begin{abstract}
Array configurations built on a hierarchy of simple elements have excellent properties for crosscorrelation imaging interferometers including a smooth distribution of measured Fourier components, high angular resolution, low side lobes, and compact array size. Compared to arrays with a Gaussian distribution of antenna separations, hierarchical arrays (H-arrays) produce beams with higher angular resolution and a tighter concentration of the total power (encircled energy) within a smaller area around the main beam. An attractive feature of $\mathrm{H}$-arrays is their simplicity. The relationships between the Fourier coverage and the array configuration are easy enough to understand that they can be adjusted to achieve different design goals without the need for numerical optimization. H-arrays will be useful for future multi-element interferometers.
\end{abstract}

Keywords: Instrumentation: interferometers, telescopes, techniques: interferometric.

\section{Introduction}

The design or configuration of a cross-correlation imaging interferometer, the placement of the antennas, presents a challenge because the properties desired for imaging cannot all be optimized simultaneously. Imaging interferometers such as those used in radio astronomy construct a picture of the sky from a set of measured Fourier components. Each pair of antennas in a multi-antenna array measures one Fourier component whose scale or spatial wavelength is proportional to the distance between the pair and whose direction is aligned with their orientation. The entire set of Fourier components is given by all the possible pairs, and the point source response or the beam of the telescope is given by their Fourier transform. Thus the relative locations of the antennas determines the quality of the images obtained by the telescope (Bracewell, 1958).

The impossibility of obtaining an infinite set of Fourier components requires choices as tradeoffs among different qualities desired for imaging. For example, the extent or size of the array should be small because the atmospheric phase coherence decreases with distance and also because the construction is easier. At the same time, the width of the beam or point-source response should also be small to achieve high angular resolution. Because the sizes of the array and the beam are related through the Fourier transform of the antenna separations, these two goals amount to minimizing both the extent of a function and the extent of its Fourier transform. The challenge is that the two generally have an inverse relationship, one big the other small. Another challenge lies in the trade-off between the angular resolution and the level of the side lobes around the main beam. The highest angular resolution requires as many long spatial wavelength Fourier components (antenna separations) as possible. This requires a sharp cut-off in the number of components at the maximum spatial wavelength (antenna separation) because a smooth transition to the maximum necessarily implies a decreasing number of components nearing the limit. The sharper the transition, the larger the side lobes so that generally, the higher the angular resolution, the higher the side lobe levels. A further design goal is complete sampling of the Fourier plane. Although imaging requirements 
might push the distribution to emphasize shorter or longer wavelength spatial frequencies, gaps in the distribution represent missing information and allow unfaithful imaging. The assumption is that the likelihood of a significant error in the estimate of a Fourier component increases with the distance in Fourier space from the component to the measurement. Therefore, the best estimate of the strength of a Fourier component is a nearby measurement, and the distribution of separations should minimize the maximum distance from any point in the Fourier plane to the nearest measurement.

In summary, four design goals are:

(1) High angular resolution

(2) Concentrated power, low side lobes

(3) Compact array size

(4) Uniform or smooth distribution of Fourier components

How do we design arrays to fulfill these goals? How do we measure success? Do the trade-offs define a continuous space with the different goals at the vertices of a polyhedral boundary? Can we position our design within this space to best meet the imaging requirements of a particular application? Must we rely on numerical optimization that might provide a configuration but no explanation leaving doubt that we have found the best possible design?

This paper introduces array configurations built on a hierarchy made by repeating one simple array configuration on different scales. In this construction, the relationships between the antenna locations, their separations, and the beam are simple enough to provide answers to these questions. For example, hierarchical or H-arrays can be designed to achieve either higher angular resolution or a more concentrated beam with lower side lobes by simply changing the scaling between the levels of the hierarchy to emphasize shorter or longer spatial wavelength Fourier components. Because H-arrays are nested and scaled, the choice can be made in the design stage by scaling the separation between the hierarchies, or for arrays designed with more possible locations than antennas, the beam can be varied by populating different levels of the hierarchy. This makes H-arrays particularly well suited for the strategy employed by most radio astronomy interferometers of periodically rearranging a smaller number of antennas among a larger number of locations to observe different angular scales. H-arrays will be useful for future interferometers with many antennas.

\section{Classic Pairs}

Two well-known Fourier transform pairs (Bracewell, 1999) illustrate the trade-offs and suggest that there is a functional form for a continuously varying aperture distribution resulting in a beam pattern that can be varied smoothly between the design goals. For the moment, suppose that we have complete control over the aperture distribution, and can assume an ideal continuous distribution. We will come back to the question of how an array can be configured to provide the required aperture.

First, consider a uniform distribution across the aperture. In one-dimension this would be a boxcar function or in two dimensions a uniform disk. The beam pattern is the Fourier transform of the aperture distribution, which for one and two dimensions respectively, is the sinc function, $\sin x / x$, and the function $J_{1}(r) / r$ where $J_{1}$ is a Bessel function. In the two-dimensional case, the power pattern, or the square of the beam, is the Airy function. The beam and power patterns are shown in Fig. 1. The angular resolution of the beam power is $\lambda / D$, the wavelength of the observing frequency divided by the diameter of the aperture. For example, if the aperture is $1000 \mathrm{~m}$ in diameter and the observing frequency is $230 \mathrm{GHz}$. Then the angular resolution, measured by the width of the power in the main beam, is 0.27 arc seconds, fullwidth at half-maximum (FWHM). However, a significant amount of the power is outside the main beam in the side lobes. The encircled energy, or the percentage of the total beam power as a function of radius is a measure of the concentration of the power in the main beam. The beam of a $1000 \mathrm{~m}$ uniform aperture at $230 \mathrm{GHz}$ contains $98 \%$ of the power within a radius of 1.53 arc seconds.

In contrast, consider a Gaussian distribution whose Fourier transform is also a Gaussian with an inverse relation in widths, $\sigma$ and $\lambda / \sigma$. Because the Gaussian has no side lobes other than its own extended wings, all the power is concentrated in the main beam. However, the inverse relation between the widths in the real and Fourier domains means that a large array size is required to achieve a small beam size. Furthermore a Gaussian aperture must be truncated at a finite radius. A Gaussian aperture of $1000 \mathrm{~m}$ diameter with a width of one-half the radius or $\sigma=250 \mathrm{~m}$ has an angular resolution 

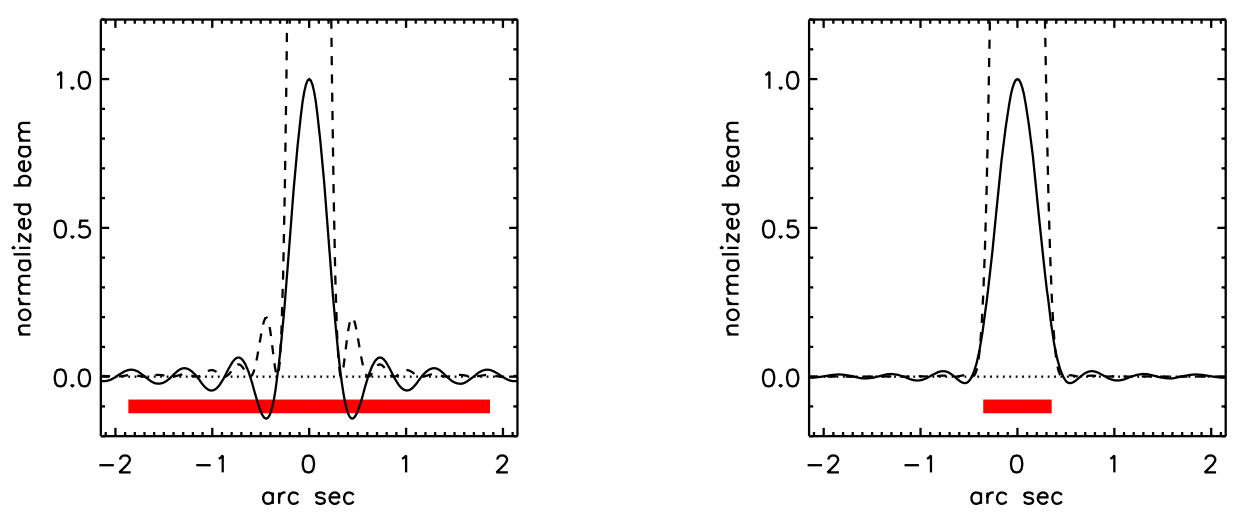

Fig. 1. (Color online) Left: One dimension of the beam (solid line) and the beam power (dashed line) of a uniform circular aperture. The beam pattern is normalized to one. The beam power is multiplied by 10 so that the scale shows the percentage of the peak power. The scale in arc seconds assumes a hypothetical uniform aperture of $1000 \mathrm{~m}$ diameter and an observing frequency of $230 \mathrm{GHz}$. The main beam is relatively narrow, 0.27 arc seconds FWHM. Because of the high side lobes, the radius that contains $98 \%$ of the total power is relatively wide, 1.53 arc seconds, indicated by the red horizontal bar. Right: One dimension of the beam and power pattern of a 2D Gaussian aperture with $\sigma=250 \mathrm{~m}(2.35 \sigma=\mathrm{FWHM})$ truncated at $1000 \mathrm{~m}$. The main beam is relatively wide, 0.40 arc seconds FWHM. Because the side lobes, caused by the truncation, are relatively low, $98 \%$ of the energy is encircled within a relatively narrow radius of 0.34 arc seconds. The figures of merit are listed in Table 4 .

of $\lambda / \sigma=0.40$ arc seconds. This is significantly broader than the beam of a uniform aperture, but the encircled energy of the Gaussian is more concentrated. The radius encircling $98 \%$ of the total power in 2.15 arc sec is 0.35 arc seconds. The beam and power patterns are shown in Fig. 1. The figures of merit are listed in Table 4.

These two Fourier transform pairs illustrate two different trade-offs: first, between the angular resolution and the size of the array; and second between the angular resolution and the encircled energy which is sensitive to the amount of power in side lobes. In general, the best apertures are a compromise between these two extremes, a uniform distribution with a smoothed or apodized boundary.

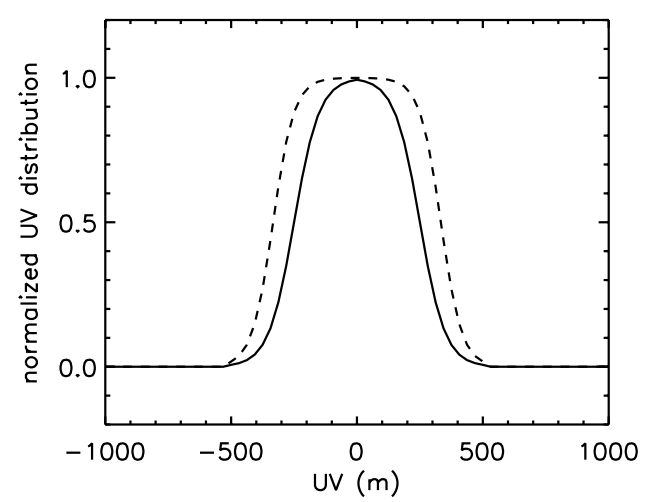

For example, a sigmoid such as the logistic function $y=1 /(1+\exp (-(x-a) / b))$ defines a curve which is uniform, equal to one in its interior, and has a smooth boundary at $a$ whose transition width, $b$, can be continuously varied. Figure 2 (left) shows two aperture distributions made with the logistic function with different values for the width of the boundary, $b=50$ and $42 \mathrm{~m}$, and its location, $a=250$ and $333 \mathrm{~m}$. Both apertures are truncated at a radius of $500 \mathrm{~m}$. Both beams (Fig. 2 right) have broader FWHM and lower side lobes than the beam of the idealized uniform aperture with a sharp boundary (Fig. 1). The comparison here shows additionally that the side lobes decrease with the increasing width or smoothness of the boundary

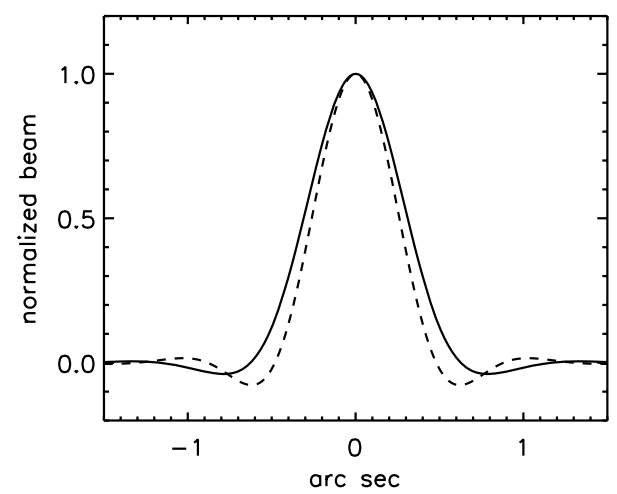

Fig. 2. Left: Two examples of a uniform distribution with a smoothed boundary using the logistic function with different constants to create two different boundary widths. Right: Beam patterns corresponding to these two aperture distributions. The comparison shows that the side lobe levels decrease with the smoothness of the distribution while the beam width increases and the angular resolution decreases. 
while the beam width increases, and the angular resolution decreases.

Finally, we must come back to the constraint that the aperture distribution is not arbitrary but given by the separations of the individual antennas. The relationship between the pattern of antenna locations and the pattern of their separations is not always obvious. Particularly with Earth rotation synthesis (ERS) (Ryle, 1962), it is a challenge to arrange the individual antennas to create a desired distribution from the sum of their separations. The fourth design goal indicates how well the antenna separations approximate a continuous distribution.

\section{Previous Array Designs}

One of the first arrays for radio astronomy, the Mills cross (Mills \& Little, 1953) had regularlyspaced dipole antennas aligned in the shape of a cross which provided a square aperture with a regularly-spaced, two-dimenensional grid of Fourier components. Ryle \& Hewish (1960) developed an interferometer with more sampling efficiency by eliminating one of the arms, which changed the cross to a T-shape, and by locating the antennas with non-uniform spacing in such a way as to minimize the number of separations of the same length. Minimum redundancy is an advantange particularly for interferometers that use expensive parabolicdish antennas rather than dipoles. The theory of minimum redundancy was further explored by Moffett (1968) and extended to a large number of antennas by Ishiguro (1980). Interferometers such as the Hat Creek Observatory/BIMA (Welch et al., 1996), the Owens Valley Radio Observatory Millimeter Array (Padin et al., 1991), and the Plateau de Bure Interferometer (Guilloteau et al., 1991), among others, are T-shaped arrays designed for Fourier sampling with minimum redundancy.

The Very Large Array (VLA) (Thompson et al., 1980) improves on the T-shape by arranging the 3 arms in a Y-shape which provides better sampling when used in ERS. A typical astronomical observation is about 8 hours, or one-third of a complete rotation. The antennas are distributed along the arms according to a power-law spacing. This creates a non-uniform sampling that allows a higher dynamic range between the smallest and the largest measured Fourier component. In contrast, the Submillimeter Array interferometer is designed to provide uniform and non-redundant Fourier sampling for high image fidelity (Keto,
1997). Uniform coverage is discussed more immediately below (Sec. 4) and power-law spacings in Sec. 9.

Yet another design goal, a Gaussian-shaped beam, was used to design the configurations for the Combined Array for Research in Millimeter Astronomy (CARMA) (Helfer, 2004). The large number of antennas of the recently built Atacama Large Millimeter Array (ALMA) (Wooten \& Thompson, 2009) allows more flexibility to shape the beam, and there are three different design goals for configurations of different scale. The most compact configuration seeks to minimize side lobe levels, the intermediate is a spiral pattern with a Gaussian beam in mind, and the most extended is a "Y" shape modified to reduce the side lobes of the beam (Conway, 2006; Holdaway, 2007). Examples of the three configurations are shown in Sec. 14.

The Long Wavelength Array (LWA) distributes 256 antennas in a uniform random pattern that is modified to reduce the side lobe levels (Kogan, 2000). Random arrays are discussed in Sec. 10.

One proposed design for the Square Kilometer Array (SKA) puts the antennas along logarithmic spirals and seeks to minimize large gaps in the Fourier sampling (Millenaar et al., 2011). Spiral arrays are discussed in Sec. 9 and the minimum gap criterion in Sec. 4.2.

The study presented here in this article does not discuss the merits of different design goals. These depend on the particular scientific aims of the observatories and their particular constraints, for example, the number of antennas. The different designs of the observatories listed above represent a significant diversity of goals, and the list does not even include all radio astronomy arrays. Rather, this paper shows how array design can be understood in terms of trade-offs between different goals and how the design process can be simplified by a hierarchical strategy that allows the designer to place the array at a desired point between the trade-offs.

\section{Uniform Sampling by Curves of Constant Width}

The hierarchical arrays introduced in this paper are constructed by repeating a simple configuration, a subarray, on multiple scales. A good choice for the repeating pattern is one of the configurations that approximates uniform sampling in the Fourier plane. This property ensures that for each scale 
the gaps in the Fourier coverage, or more precisely the separations between sampling points are all about the same size. Since they are a basis for the $\mathrm{H}$-arrays, it is worth reviewing the properties of the configurations that provide the best uniform coverage.

A previous paper showed that arrays designed on the figures known as curves of constant width (CW) come closest to approximating a uniform aperture distribution (Keto, 1997). These figures are in the shape of closed rings. The name, constantwidth, refers to the diameter. These curves have the property that from any point on the curve, the maximum distance to the opposite side, effectively the diameter, is always the same. This guarantees that the boundary of the aperture and its Fourier transform, the beam, are both circular. For example, the circle, which by definition has a constant diameter, is a curve of constant width. The circle can be thought of as a CW-curve with an infinite number of sides. The Reuleaux triangle is the limiting case of a CW-curve with the least (three) number of sides. Of the two, the Reuleaux triangle is preferred for array design and was adopted for the design of the Submillimeter Array.

The uniform aperture distribution with its $J_{1}(r) / r$ beam approximated by a $\mathrm{CW}$-array lies toward one end of our space of design tradeoffs accomplishing three of the design goals, high angular resolution, compact array size, and uniform sampling but at the expense of rather high side lobes. The uniform sampling has another attractive feature. In imaging applications, the uniform sampling allows the measured Fourier components to be combined with equal weights ("natural weighting" in radio astronomy) and therefore achieves the highest sensitivity simultaneously with uniform sampling. CW-arrays are a good choice for interferometers that have relatively few antennas and therefore deeply cherish each individual Fourier component and regret weighting down any of them. Furthermore, a small number of antennas limits the flexibility to shape the beam, and the small number of measured Fourier components necessarily results in high side lobes which, being unavoidable, are therefore less of a design concern. Imaging with high side lobes relies on numerical techniques to deconvolve the beam pattern from the image (Hogbom, 1974).

$\mathrm{CW}$-arrays are also the best choice for dithering patterns for flat-fielding multi-pixel imaging arrays
(Arendt et al., 2000). In this application the goal is to measure the relative sensitivity of all the pixels in a sensor array such as a CCD. One possibility is to point the telescope repeatedly until each pixel has viewed the same patch of sky. This requires many observations, one for each pixel in the array, but each comparison to determine the relative sensitivity of two pixels requires the use of only two measurements. Another possibility is to point the telescope only twice in such a way that the view is shifted by one pixel. Each pixel then views the patch of sky previously viewed by its neighbor, and the sensitivity of neighboring pixels may be measured relative to each other. The relative sensitivity of all the pixels is found by working across the array comparing neighboring pixels. This requires only two observations, but the number of comparisons needed to determine the sensitivity of any two pixels increases with their separation. The noise in each intermediate measurement and comparison reduces the accuracy of the flat-fielding. The optimal strategy uses as few observations and as few intermediate comparisons as possible. This is achieved by distributing the pointing shifts uniformly across the space of all possible shifts, exactly analagous to the problem of distributing the separations of antennas uniformly across their separation space. In their study, Arendt et al. (2000) objectively compared a number of different array designs and verified that the $\mathrm{CW}$-arrays provide the best approximation to uniform sampling. The Spitzer Space Telescope uses an observing pattern based on the Reuleaux triangle for imaging with its infrared cameras.

\subsection{A 6-element $C W$-array}

The simplest example of $\mathrm{CW}$-array is the sixelement pattern in Fig. 3 whose separations are uniformly distributed on an hexagonal grid. The antenna coordinates are given in Table 1 . Visually there appears to be a hole in the center that is missing a separation. This is a separation of zero, or equivalently the Fourier component of zero spatial wavelength corresponding to the total power. All interferometers are missing this measurement of total power. Figure 3 shows that the distance between all the separations, including the distance from the smallest separations to the zero point, is exactly one. In this sense, the configuration is not missing short spacings. The shortest spacing is set by the distance between the pairs of antennas 

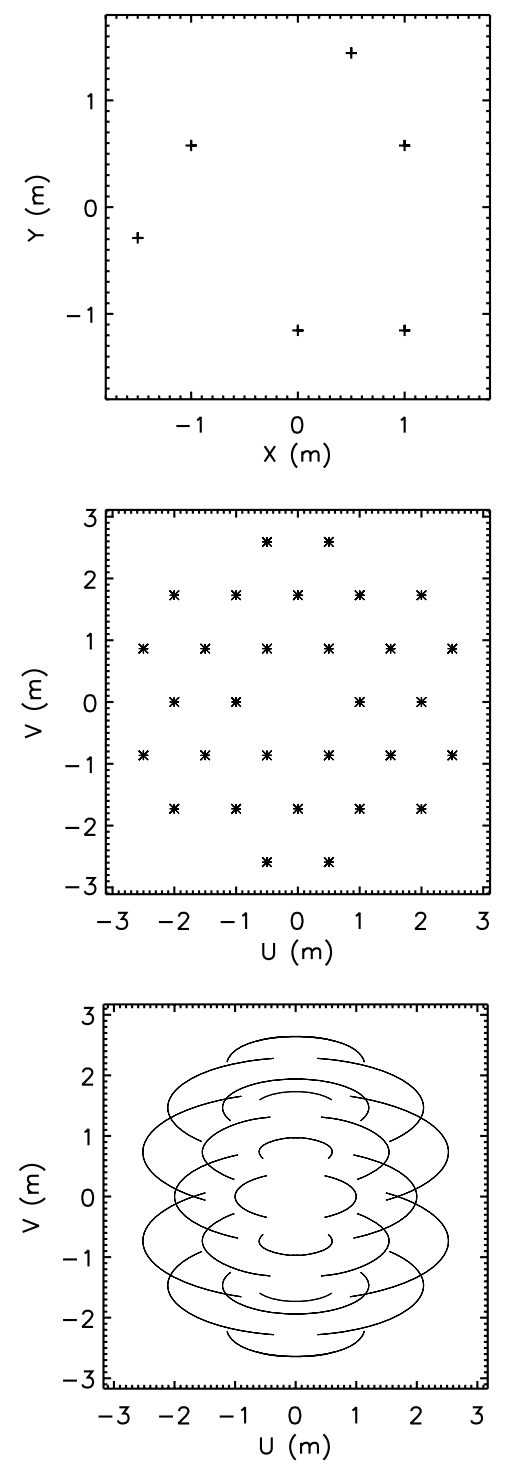

Fig. 3. Top: Antenna locations of a simple 6-element array whose separations (UV coverage) (middle) are uniformly spaced on a hexagonal grid. Bottom: Tracks of the antenna separations (baselines) in an $8.2 \mathrm{hr}$ Earth rotation synthesis (ERS). The uniform distribution of separations (middle) produces a reasonably uniform distribution of baseline tracks.

on each side of the Reuleaux triangle which of course can be made arbitrarily small. The reason the central hole in the coverage looks larger in this array than in other arrays with non-uniform coverage is because the uniform coverage limits the size of the largest separation. For example, arrays with a power-law distribution of antenna separations achieve a higher ratio of the largest to smallest separation, resulting in higher spatial dynamic range, but only by leaving larger holes in the coverage at larger spatial wavelengths. However, in these nonuniform arrays, the central hole looks smaller with
Table 1. Antenna positions for 6-element subarray.

\begin{tabular}{cc}
\hline$X$ & $Y$ \\
\hline 0 & 0 \\
1 & 0 \\
1 & $\sqrt{3}$ \\
$1 / 2$ & $3 \sqrt{3} / 2$ \\
-1 & $\sqrt{3}$ \\
$-3 / 2$ & $\sqrt{3} / 2$ \\
\hline
\end{tabular}

respect to the total extent of the non-uniform coverage. The next section, Sec. 7, shows that one advantage of hierarchical arrays is that this central hole can be filled by a smaller scale in the hierarchy.

While the UV coverage in ERS is no longer strictly uniform, the mid-points of the ERS tracks are still on the original uniform grid. This means that arrays that have good snapshot coverage generally have good coverage in ERS even though the coverage in snapshot and ERS is not the same. Figure 3 shows the tracks of the separations (baselines in radio astronomy) in an $8.2 \mathrm{hr}$ ERS assuming the array is located at a latitude of $23^{\circ}$ and the target transits through the zenith. As shown in the figure, ERS generates a pattern of tracks, or an effective aperture, slightly larger in extent in the north-south direction than east-west. This is because the target is low on the horizon at the beginning and end of the track when it is rising and setting. At these times from the vantage point of the target, the array and its antenna separations appear shortened by projection. This results in UV coverage that is narrower and a beam pattern that is wider in the east-west direction than north-south. It is trivial to make the beam circular by adjusting the aspect ratio of the array, but the correction is also a function of the declination of the target.

\subsection{The minimax metric}

$\mathrm{CW}$-arrays produce the most uniform sampling across a circular aperture, but aside from the particular 6-element pattern in snapshot observing (discussed above), their separations do not lie exactly on a uniform grid. How do we measure uniformity? For imaging arrays, the most important feature of uniform coverage is that no part of the relevant Fourier space is very far from a measurement. This decreases the possibility of error which increases with the distance in Fourier 

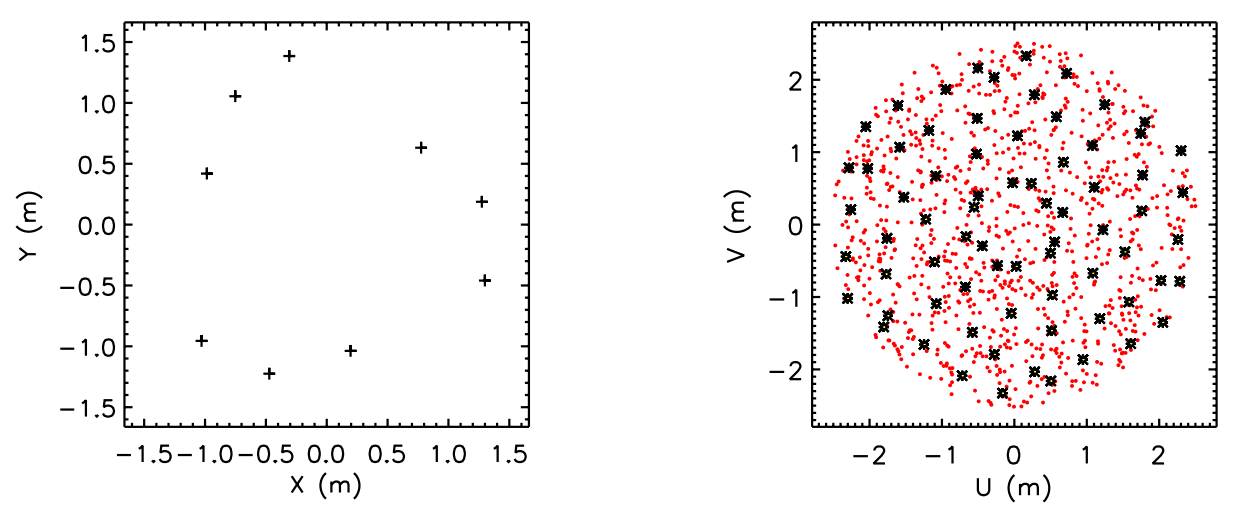

Fig. 4. (Color online) Left: Antenna locations of a 9-antenna array whose separations approximate a uniform distribution. Right: Minimax test. Crosses show the antenna separations and red dots show the random UV points generated by a Monte Carlo algorithm.

space between the component and a nearby measurement, assuming that there is some smoothness or coherence in the image itself. With this in mind, uniformity can be defined in a statistical sense with a particular figure of merit, a minimax that measures the maximum separation from every point in the Fourier plane to the closest measured Fourier component. With the usual distance as the metric, the best approximation is the one that minimizes the maximum distance, $\min \sum\left(\max _{i}\left[\left(U-u_{i}\right)^{2}+\right.\right.$ $\left.\left.\left(V-v_{i}\right)^{2}\right]\right)$ where $U$ and $V$ are probability densities that represent the desired distribution across the entire Fourier plane within the circular boundary allowed by the maximum antenna separation, and $u_{i}$ and $v_{i}$ are the coordinates of each antenna separation. A particularly simple way to compute this figure of merit is with a Monte Carlo algorithm. To measure how closely the separations of an array approximate a uniform distribution, generate a set of random points $U, V$, uniformly distributed inside the boundary of separations and compute the distance between all pairs $U, V$ and $u_{i}, v_{i}$. The best approximation minimizes the maximum distance or the average maximum distance. Figure 4 illustrates the minimax for a 9-antenna array. The antenna locations for this array are listed in Table 2. In the right panel the antenna separations are shown as asterisks and one particular realization of a set of random UV points is shown as red dots. An actual evaluation would use many more random points than the ones shown here for illustration. The number of UV points necessary for an accurate measure is about 10 to 100 times the number of separations. It is easy to determine the accuracy of the algorithm by running a few trials with different numbers of points and different realizations of the
Table 2. Antenna positions for 9-element array.

\begin{tabular}{cc}
\hline $\mathrm{X}$ & $\mathrm{Y}$ \\
\hline-1.02847 & -0.955366 \\
-0.471921 & -1.22493 \\
0.195772 & -1.03746 \\
1.29924 & -0.459679 \\
1.27441 & 0.187321 \\
0.775329 & 0.631558 \\
-0.308142 & 1.38484 \\
-0.751473 & 1.05465 \\
-0.984755 & 0.419072 \\
\hline
\end{tabular}

random distribution. This figure of merit was used in (Keto, 1997) to show that the arrays that best approximate a uniform distribution of separations are based on the Reuleaux triangle. The minimax figure of merit is different from other figures of merit that consider the antenna separations only with respect to one another.

\section{Figures of Merit}

What if we want a more concentrated beam and power pattern with lower side lobes and a smaller radius of encircled energy than provided by a uniform aperture distribution? Hierarchical arrays are one solution. First we need a complete set of figures of merit to quantitatively evaluate different array designs in terms of each of the four design goals. The first two goals can be combined into the product of the array size and the beam size, leaving three.

(1) $K_{n n}=$ maximum diameter of the array times the diameter that contains $n n$ percent of the encircled energy. This measures the degree to 
which the design is compact in both real and Fourier space. Because the example arrays all have a maximum separation of $1000 \mathrm{~m}$ the $K$-product is sensitive to the distribution of the power between the main beam and the side lobes. For radio frequency arrays of $1000 \mathrm{~m}$ with arc second resolution, the units are meter arc seconds. This measurement depends on the angular size scale used to define $100 \%$ of the total power. Since there are a limited number of Fourier components, they never completely cancel, and the side lobes are infinite in extent. A good practice is to integrate out until the decrease in side lobe level slows. For our example arrays of $1000 \mathrm{~m}$ diameter and observing frequency of $230 \mathrm{GHz}$, we use a radius of 2.15 arc seconds.

(2) The angular resolution, the full width at half maximum (FWHM) of the main beam. We use the root of the product of the widths in the north-south and east-west directions.

(3) A smooth distribution of UV points. There are several ways to measure the smoothness. Some experimentation shows that a simple and satisfactory measure is the variance off a polynomial fit to the density of antenna separations as a function of separation. We use a third order polynomial and the reduced $\chi^{2}$ of this fit.

To generate the figures of merit, the hypothetical observatory is located at a latitude of $23^{\circ}$ tracking a source through zenith in Earth rotation synthesis. The UV tracks are calculated for $8.2 \mathrm{hrs}$ between Hour Angles \pm 4.1 with points recorded every $0.25 \mathrm{hrs}$. The UV tracks and the beams are calculated assuming that the arrays have a maximum diameter of $1000 \mathrm{~m}$, and that the observing frequency is $230 \mathrm{GHz}$.

The maps of the beam pattern are made with a particularly punishing color scale that emphasizes the low-level side lobes. The beam is normalized by its peak, then subject to an asymmetric sigmoid function, the Gompertz function $y=\exp (-\exp (-a x))$ with constant, $a=50$, large enough to saturate the beam at a few percent revealing the side lobes. The color table, inspired by Tang sancai (three colors) pottery, shows the negative and positive side lobes in blue and red. The asymmetry of the Gompertz function compensates in part for the visual bias that red appears slightly brighter than blue.

\section{Hierarchical Configurations}

To construct a hierarchical array, repeat the subarray pattern on a larger scale with copies of the subarray distributed on a pattern equal to itself. If we call the first level the s-level, the second the p-level, the third the d-level, and the fourth the f-level, a two-level array with 6 elements in the first level and 6 in the second is indicated by s6p6. Figure 5 shows this configuration with the second level scaled to 12 times the first. The two hierarchies of antennas create the two hierarchies of separations. In this example, the scaling between the hierarchical levels is large enough that the separations in different levels do not overlap.

In the very center marked in green are the $30 \times 6$ shortest separations within each of the 6 subarrays. To eliminate the redundancy in the separations that would occur if the subarrays were identical, the subarrays are rotated and scaled very slightly with respect to each other. Relative to the subarray at the bottom of the figure, the subarrays are rotated by $50,10,40,20$, and 30 degrees moving counterclockwise around the pattern, and scaled by factors of 1.03, or from the bottom, by 1.03, 1.06, 1.09, 1.13, and 1.16. This produces 180 unique short separations rather than 6 copies of the same 30 separations (Fig. 5 top right). The entire pattern is rotated 60 degrees with respect to the 6 positions in Table 1.

The second level of the hierarchy of separations is made up of all possible separations of the antennas of different subarrays. These larger scale separations show the same uniform pattern as in Fig. 3, but with patches made of the 30 possible pairs of the 6 subarrays instead of the $30 \mathrm{UV}$ points from the 6 individual antennas. In each of the 30 patches there are 36 points from the 36 possible pairs of antennas between 2 subarrays. Figure 5 (middle right) shows one set of these 36 separations. Each patch has a slightly different pattern. Finally, Fig. 5 (bottom) shows how ERS drags each of the 30 patches across the UV plane like a wide paint brush across a white canvas. Because the separations of the 6-element subarray are uniformly distributed, so are the midpoints of the tracks of the second level of the hierarchy.

The extension to further hierarchical levels is obvious. The entire two-level array is repeated on the same basic pattern of the subarray. To keep the figures from becoming too crowded, the following examples refer to just two or three levels. 

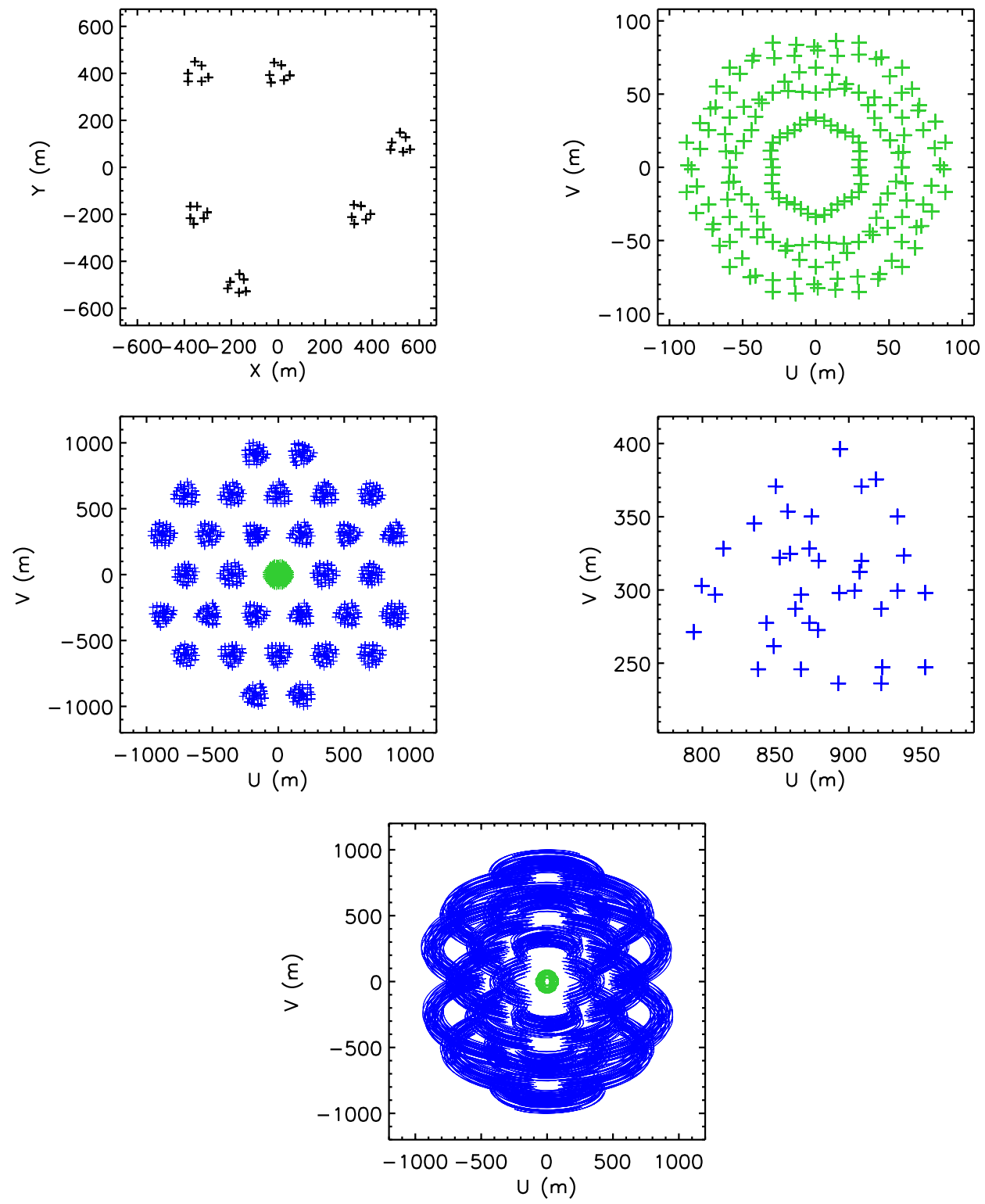

Fig. 5. (Color online) Top left: Antenna locations in a 2-level s6p6 array. The subarrays are rotated relative to the subarray at the bottom of the figure by 50,10,40,20, and 30 degrees moving counterclockwise around the pattern. The subarrays are also scaled by factors of 1.03, or from the bottom, by 1.03, 1.06, 1.09, 1.13, 1.16. The entire pattern is rotated 60 degrees with respect to the 6 positions in Table 1. Middle left: Antenna separations. The $180=30 \times 6$ short separations between antennas within each of the 6 subarrays occupy the center of the UV plane in green and in detail top right. The 30 other patches around the center are the separations between the subarrays. The set of patches replicate the uniform spacing of the basic 6 -element array (Fig. 3). Detail of one patch middle right. The exact pattern is different in each patch. (bottom) UV tracks in an $8.2 \mathrm{hr}$ ERS. Each of the 30 patches moves across the UV plane like a paint brush.

\section{The Flexibility of a 2-Level H-Array}

The simple 36-element 2-level hierarchy pattern can be scaled to select a point source response along the continuum of choices in the trade-off between high angular resolution and low side lobes. First, to design a high angular resolution beam, scale the second level just large enough to place the 30 patches of separations side-by-side. Next, to design a beam with low side lobes, scale the second level so that the patches overlap and create a distribution that tapers gracefully to the boundary.

\subsection{A high angular resolution beam}

Scaling the second level by a factor of 5.5 relative to the first (Fig. 6 top left) produces a tiered distribution of separations with a nearly uniform density 

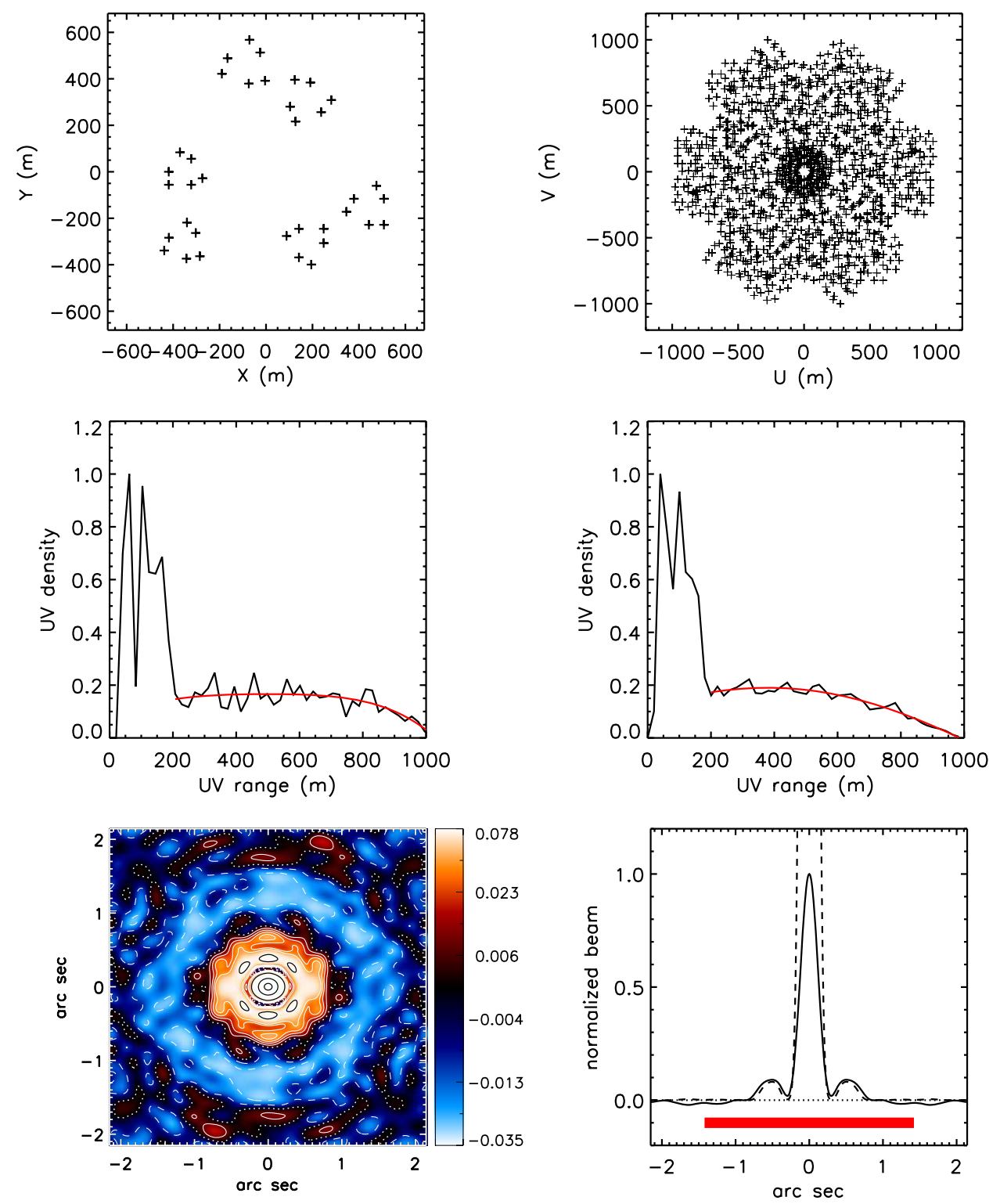

Fig. 6. (Color online) A 36-element s6p6 array designed for high angular resolution. Top left: Antenna positions. Moving counterclockwise around the figure the subarrays are rotated with respect to the left subarray of the two on the bottom by 20 , $60,30,100$, and 20 degrees. The subarrays are also scaled by factors of 1.05 with respect to each other, or counterclockwise from the left bottom, by 1.05, 1.10, 1.16, 1.22, and 1.28. Top right: Separations and UV coverage in snapshot at zenith. Middle left: Radial distribution of the density of separations in snapshot at zenith. The smooth red line shows a polynomial fit to the outer part of the distribution. The variance from this fit is a measure of the smoothness of the coverage in the outer zone. Middle right: Radial distribution of the density of separations in ERS. Bottom left: Beam pattern in ERS. The black contours are at 10, 50 , and $90 \%$ of the peak. From the peak, the second black contour shows the FWHM. The white contours are at 1,2 , and $3 \%$ of the peak. The negative contours are dashed. The zero contour is white and dotted. Bottom right: Beam and power pattern in the same format as Fig. 1. The beam power (dashed line) is multiplied by 10. The figures of merit are listed in Table 4.

in two regions. Figure 6 (top right) shows the twodimensional pattern of separations and the UV coverage obtained in snapshot imaging at zenith.

Figure 6 (middle left) shows the corresponding density of separations as a function of radius averaged over angle. In ERS, the elliptical arcs across the UV plane improve the UV coverage, particularly as a function of angle. Because the UV points of the shorter separations move more slowly across the UV plane, ERS also affects the radial distribution by increasing the density of the shorter separations. Nonetheless, the radial distribution in ERS still maintains its essential character (Fig. 6 middle right). The smooth red line shows the fit of 
a third order polynomial to the distribution in the outer zone. In snapshot the reduced $\chi^{2}$ is 0.00094 . The better UV coverage in ERS fills in gaps and improves the smoothness to 0.00017 .

The two-dimensional beam pattern in ERS (Fig. 6 bottom) shows the high side lobes characteristic of arrays with uniform UV coverage, but the angular resolution is exquisite, 0.17 arc seconds FWHM. The radius encircling $98 \%$ of the beam power is 1.38 arc seconds and the $K$-product,
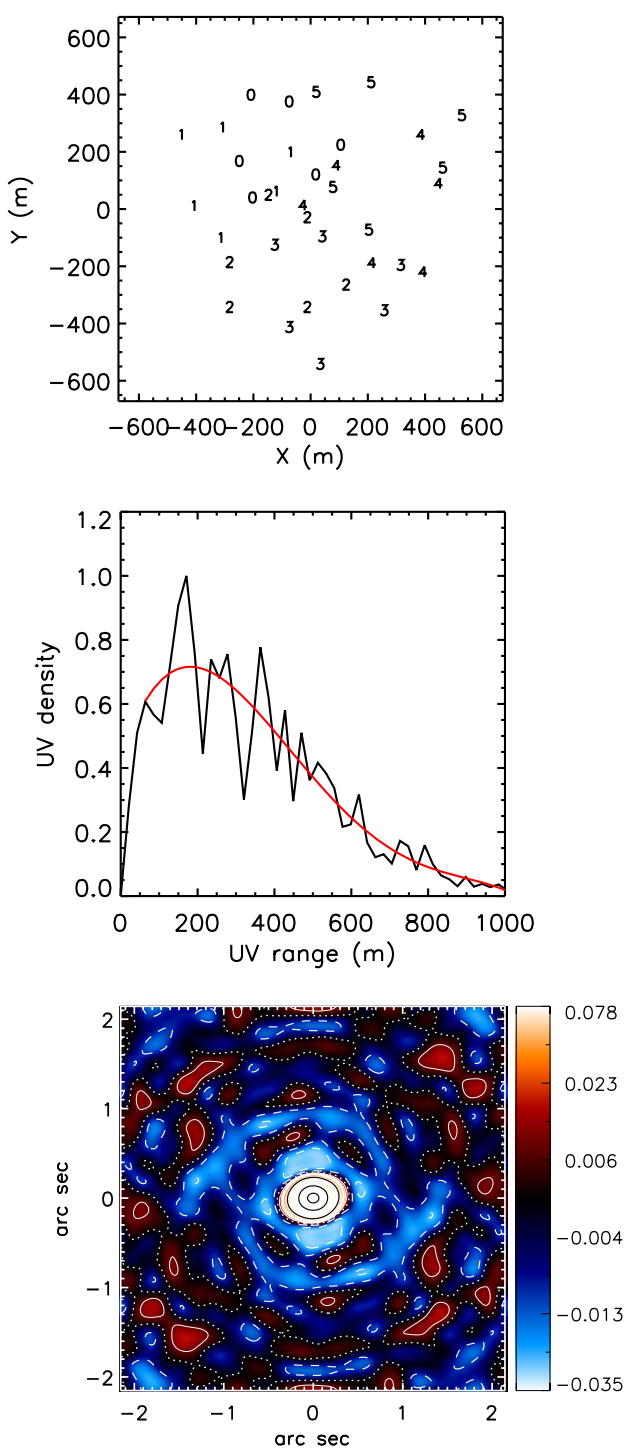

$K_{98}=1378$ (Fig. 6 bottom right). The figures of merit are listed in Table 4.

\subsection{A beam with low side lobes}

To reduce the side lobes, reduce the scaling of the second level of the hierarchy to pull the 30 patches of separations inward until they are overlapping and create a smoothly tapered distribution. Figure 7
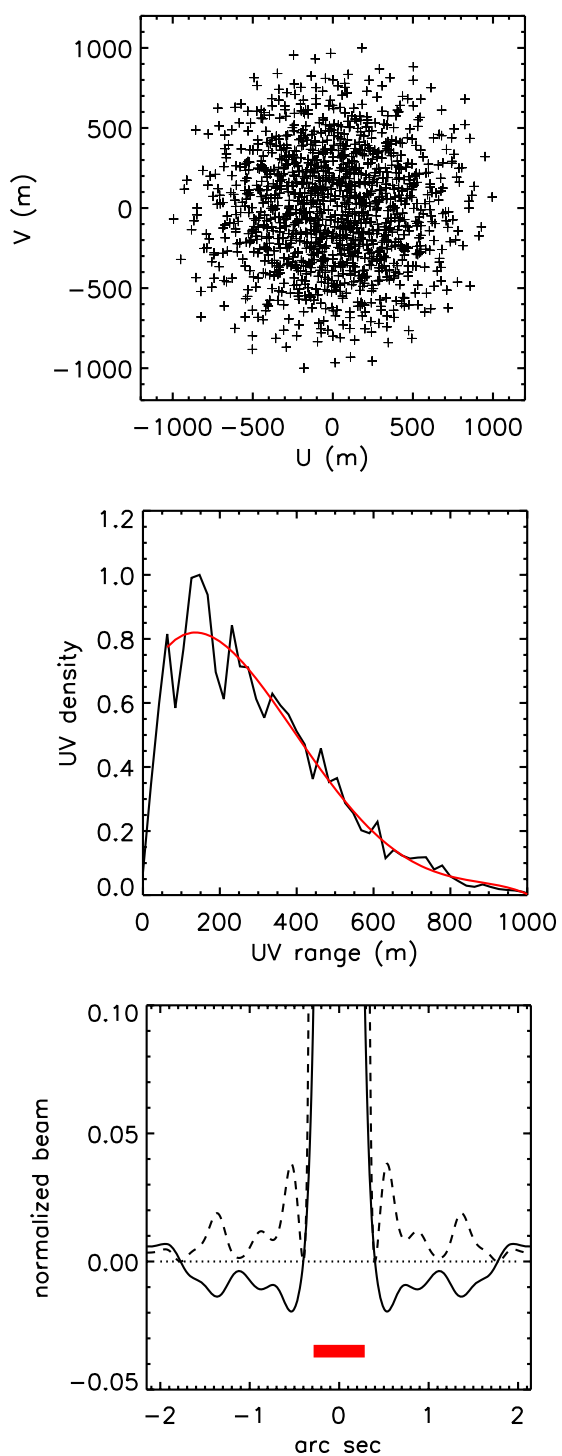

Fig. 7. A 36-element s6p6 array designed for a high concentration of power in the main beam and low side lobes. Top left: Antenna positions. The 6 subarrays overlap and each is drawn with a different number. The 6 positions marked with the number 0 belong to the same subarray. Moving counterclockwise around the figure, relative to the subarray marked by zeroes, the subarrays are rotated by 40,20,60,20,100, and 20 degrees. The subarrays are also scaled by factors of 1.075 with respect to each other, or counterclockwise from the upper left by $1.075,1.163,1.24,1.34,1.44$. The entire pattern is rotated 30 degrees with respect to the locations in Table 1. Same format as Fig. 6 except for the panel in the lower right. Here the solid line shows the bottom of the beam which is normalized to one. The dashed line shows the beam power multiplied by 100 . The scale then shows the power as a percentage of the peak power. The figures of merit are listed in Table 4 . 
shows the same plots as in the previous example but for an array with the second level only 1.5 times larger than the first. The radial distribution of the separation density is now shifted to shorter separations and gradually tapers to the maximum. The beam has lower angular resolution, 0.21 arc sec, but also lower side lobes and more concentrated beam power. The radius encircling $98 \%$ of the energy is 0.29 arc seconds, and the $K$-product is $K_{98}=285$. This beam has better angular resolution than the 0.35 arc sec Gaussian beam of our idealized example discussed in the introduction, and the smaller $K$-product indicates that the side lobes of this beam contain less energy than the extended wings of the Gaussian beams of either the truncated or idealized Gaussian apertures.

The concentrated UV distribution leaves fewer points to cover the outer zone resulting in wider gaps than in the more uniform distribution of the array in Fig. 6. The $\chi^{2}$ figure of merit measures the local smoothness and is accordingly larger even though the distribution is globally tapered to the boundary. For snapshot and ERS, $\chi^{2}$ is 0.0098 and 0.0041 , respectively.

\subsection{Design principles}

These examples show how H-arrays allow control over different scales in the UV plane. A change in the first-level subarrays affects the UV distribution of the smallest separations in the center of the UV plane and also the distribution inside the 30 patches or brush strokes in ERS of the second level but does not much affect the distribution of these patches across the UV plane. This is controlled by the placement of the subarrays in the second level. In this way we have simple and understandable control over the aperture distribution. We can shape the aperture distribution by scaling the levels to emphasize Fourier components of different scales.

The conceptual separation of scales possible with $\mathrm{H}$-arrays can be thought of as accomplishing more than one observation at the same time. Arrays for astronomical imaging are often built with multiple configurations of different sizes to measure Fourier components on different angular scales. The configurations are nested so that the separations of each configuration are just larger than the next smaller size, usually with some overlap. The full range of Fourier components is measured in multiple observations by moving the antennas to each of the configurations between observations. The multiple scales of the $\mathrm{H}$-arrays accomplish this in one observation.

\section{Sparse H-Arrays}

Is the uniform pattern of separations provided by the 6-element array of Fig. 3 essential? What if the number of available antennas is not a power of six? Two examples with a minimal subarray of 3 elements show that it is possible to obtain good coverage with sparse arrays built with a subset (half) of the basic 6-element subarray. The lower density of UV coverage leaves some gaps, but there is a positive trade-off as well. Sparse arrays can achieve a larger dynamic range, the difference between the smallest and largest separations, with fewer antennas.

\subsection{A sparse array for high angular resolution}

As with our earlier example, a larger scaling between the hierarchical levels can be used to create approximately uniform UV coverage in separate zones. Figure 8 shows an array with 3 hierarchical levels. The first two levels use 3 -element subarrays made by dropping every other element of the basic 6-element array. The third level uses the full 6 -element pattern. This array has 54 antennas in an s3p3d6 configuration (Fig. 8). The second and third levels are scaled by 3.4 and 22.0 times the basic subarray. The subarrays within the two levels are scaled by $6 \%$ and $8 \%$ to eliminate redundancy. The details describing the rotation and placement of the subarrays are listed in Table 3 . With a maximum separation of $1000 \mathrm{~m}$, the angular resolution is 0.18 arc seconds at $230 \mathrm{GHz}$. The $K$-product is $K_{98}=891$ indicating that $98 \%$ of the encircled energy is within a radius of 0.89 arc seconds. In snapshot imaging, the coverage of this s3p3d6 sparse array has less uniform coverage than the 2-level s6p6 array but still maintains the character of uniform coverage tiered in separate zones. Figure 8 shows the separations (snapshot coverage at zenith), the radial density of the separations in snapshot and ERS, and the beam and its trace in ERS. With more UV points than the 36-antenna s6p6 array, the $\chi^{2}$ measure of local smoothness is lower, 0.00027 and 0.00020 in both snapshot and ERS, respectively. The figures of merit are listed in Table 4. 

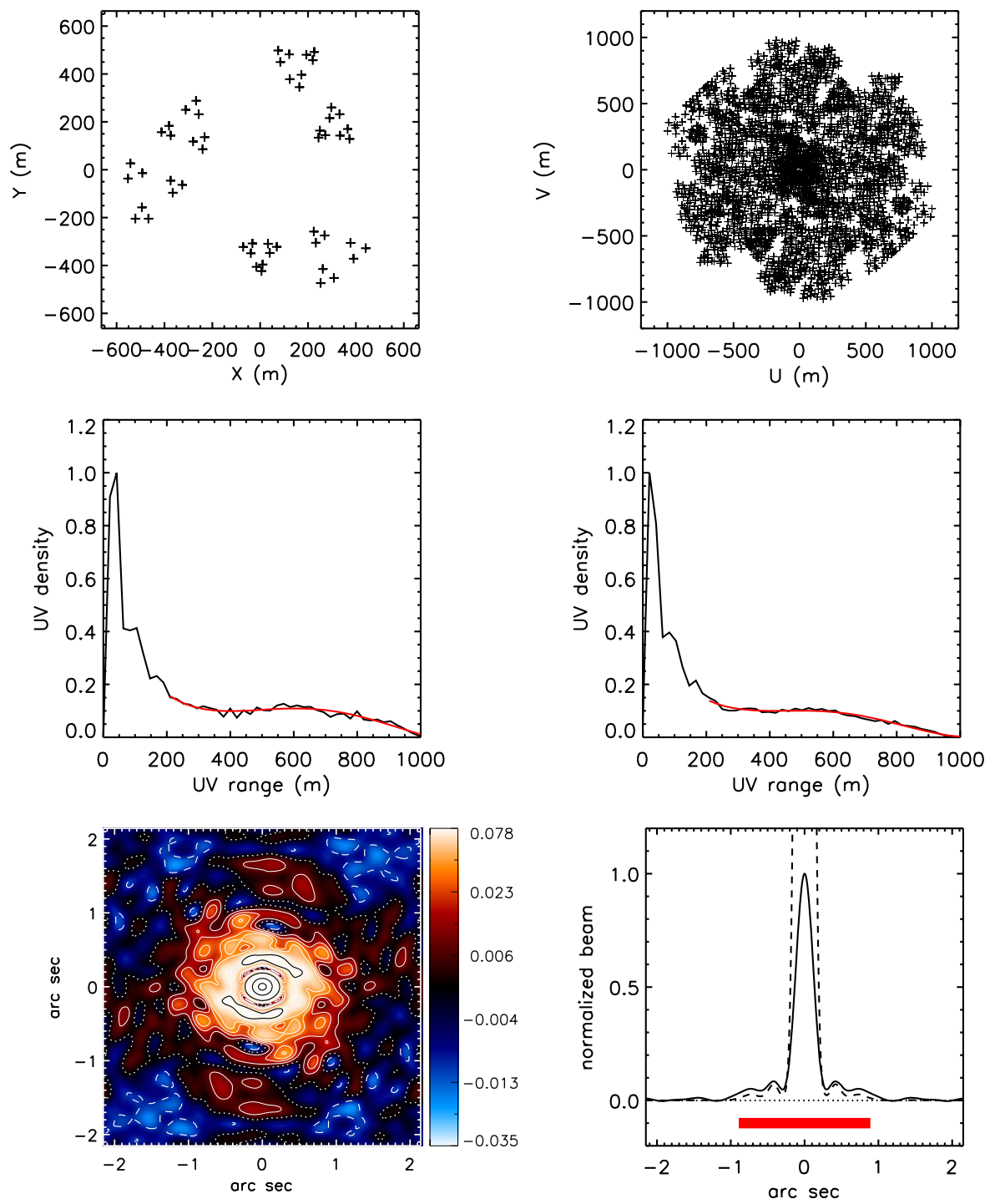

Fig. 8. Top left: Antenna locations, UV distribution, and beam for an example configuration of 54 antennas weighted toward longer baselines. Same format as Fig. 6. The rotations and scalings of the subarrays are listed in Table 3. The beam power (dashed line) in the lower right panel is multiplied by 10. The figures of merit are listed in Table 4.

\subsection{A sparse array with a concentrated beam}

Figure 9 shows an example with a factor of 2 scaling between the levels and $3 \%$ scaling of the subarrays within each level to eliminate redundant separations. The details describing the rotation and placement of the subarrays are listed in Table 3 . The smoothly tapered distribution of separations from the overlapping subarrays creates a beam with an angular resolution of 0.23 arc seconds FWHM and a $K$-product, $K_{98}=294$ indicating that $98 \%$ of the encircled energy is within 0.29 arc seconds. Both the angular resolution and encircled energy are better than the Gaussian aperture in Sec. 2. Compared to the sparse $\mathrm{H}$-array with uniform UV coverage, here the concentrated UV coverage results in less local smoothness and higher $\chi^{2}$ measures, 0.0036 and 0.0018 in snapshot and ERS respectively. The figures of merit are listed in Table 4.

\section{Hierarchical Spirals, H-Spirals}

The technique of scaling and rotating subarrays with good uniform coverage can also be used to create intelligently designed high performance spiral arrays with many antennas. These hierarchical spirals or $\mathrm{H}$-spiral arrays are aesthetically attractive and also have some practical advantages useful in construction. (The process of scaling and rotating produces spirals that are mathematically equivalent to logarithmic spirals with different constants.) 
Table 3. Subarray rotations and scalings for Figs. 8, 9.

\begin{tabular}{crc}
\hline Subarray & Rotation & Exponent of the scaling factor \\
\hline s0 1 & 100 & 0 \\
s1 & 80 & 4 \\
s2 & 60 & 1 \\
s3 & 20 & 3 \\
s4 & 20 & 2 \\
s5 & 0 & 5 \\
p0 & 0 & 0 \\
p1 & 180 & 4 \\
p2 & 60 & 1 \\
p3 & 240 & 3 \\
p4 & 120 & 2 \\
p5 & 300 & 5 \\
\hline
\end{tabular}

Notes: ${ }^{1}$ The s-level subarrays for the s3p3d6 configuration for high angular resolution shown in Fig. 8 are scaled by 1.06 raised to the power shown in the table. For the s3p3d 6 and s6p6d6 arrays shown in Fig. 9 the scaling factor is 1.03.

2 The p-level subarrays for the s3p3d6 configuration shown in Fig. 8 are scaled by powers of 1.08 . For the s3p3d 6 and s6p6d6 arrays shown in Fig. 9 the scaling factor is 1.03.

Because of their concentric rather than hierarchical structure, spiral arrays do not produce the highest angular resolution beams with a compact array size. Nevertheless, these H-spirals share the propert of the $\mathrm{H}$-arrays that a simple change in scale again moves the design between the trade-offs of high angular resolution and a more concentrated beam.

The Square Kilometer Array might be based on a spiral pattern. The primary design goal is to minimize gaps in the UV coverage within the constraints of the spiral design (Millenaar et al., 2011). The uniform coverage of the $\mathrm{CW}$-arrays by definition provides minimal gaps (Sec. 4.2). A spiral array built by scaling a CW-subarray according to a power law has larger gaps at larger scales. At each scale, the CW-subarray provides coverage with the minimal possible gaps at that scale.

\subsection{Rotation in $\mathrm{H}$-spirals}

First consider the effect of rotation. The first two examples are $\mathrm{H}$-spiral patterns based on the 9-element CW-array in Fig. 4 but with different amounts of rotation between the subarrays. The power law scaling, 1.25, is the same in both cases. The first pattern rotates the subarrays by $164^{\circ}$ to create a tight, wrapping spiral resembling a late-type galaxy (Fig. 10). In the second, a smaller rotation of $113^{\circ}$ produces a 9-ray pattern resembling the nine-armed sea stars of the Florida
Keys (Fig. 11). Both have essentially identical performance metrics. In ERS, the $\mathrm{H}$-spiral galaxy has a angular resolution of 0.23 arc seconds and a $K$-product, $K_{98}=285$. The $\chi^{2}$ measure of smoothness is 0.0029 . The H-spiral sea star has the same angular resolution, 0.23 arc seconds and a $K$-product, $K_{98}=285$, but the $\chi^{2}$ indicates that the distribution of separations is slightly smoother at 0.0026 . In snapshot, the $\chi^{2}$ measures of local smoothness for the galaxy and sea star $\mathrm{H}$-spirals are 0.0039 and 0.0024 respectively. Both these arrays have better angular resolution and tighter encircled energy than the Gaussian beam in Sec. 2. The figures of merit are listed in Table 4.

\subsection{Scaling in $\mathrm{H}$-spirals}

Differences in the amount of rotation between the levels of an $\mathrm{H}$-spiral do not much affect the properties of the beam; however, their relative scaling is quite important as the next four examples show (Figs. 12 and 13). These H-spirals are built from the basic 6-antenna pattern (Fig. 3) scaled 9 times. Different values are used in each example for the power law exponent, 1.05, 1.15, 1.25, and 1.35. The figures of merit are listed in Table 4 . At the lower value, the UV coverage and beam resemble those of a CW-array with its raptor resolution. At the high end, the scaling produces a soft beam with wide Lorentzian wings similar to the beam of VLA with its scaling factor of 1.716. However, the curved arms of the spiral produce a rounder beam with less concentrated sidelobes than the straight arms of the VLA. Similarly, amateur astronomers sometimes use curved instead of straight vanes in their Newtonian telescopes as supports for the secondary in order to spread out the diffraction pattern of the supports.

\section{Random Arrays}

Successful array designs represent a curious blend of symmetry and randomness. Configurations with too much symmetry concentrate their separations in specific patterns in the UV space resulting in the equivalent of diffraction patterns in the beam. Symmetry in circular and triangular arrays is discussed in Keto (1997) where the better performance of the triangular array is attributed to its lower degree of symmetry. Better performance for both arrays is obtained by slightly perturbing the antenna locations off of a regular distribution around the circle 

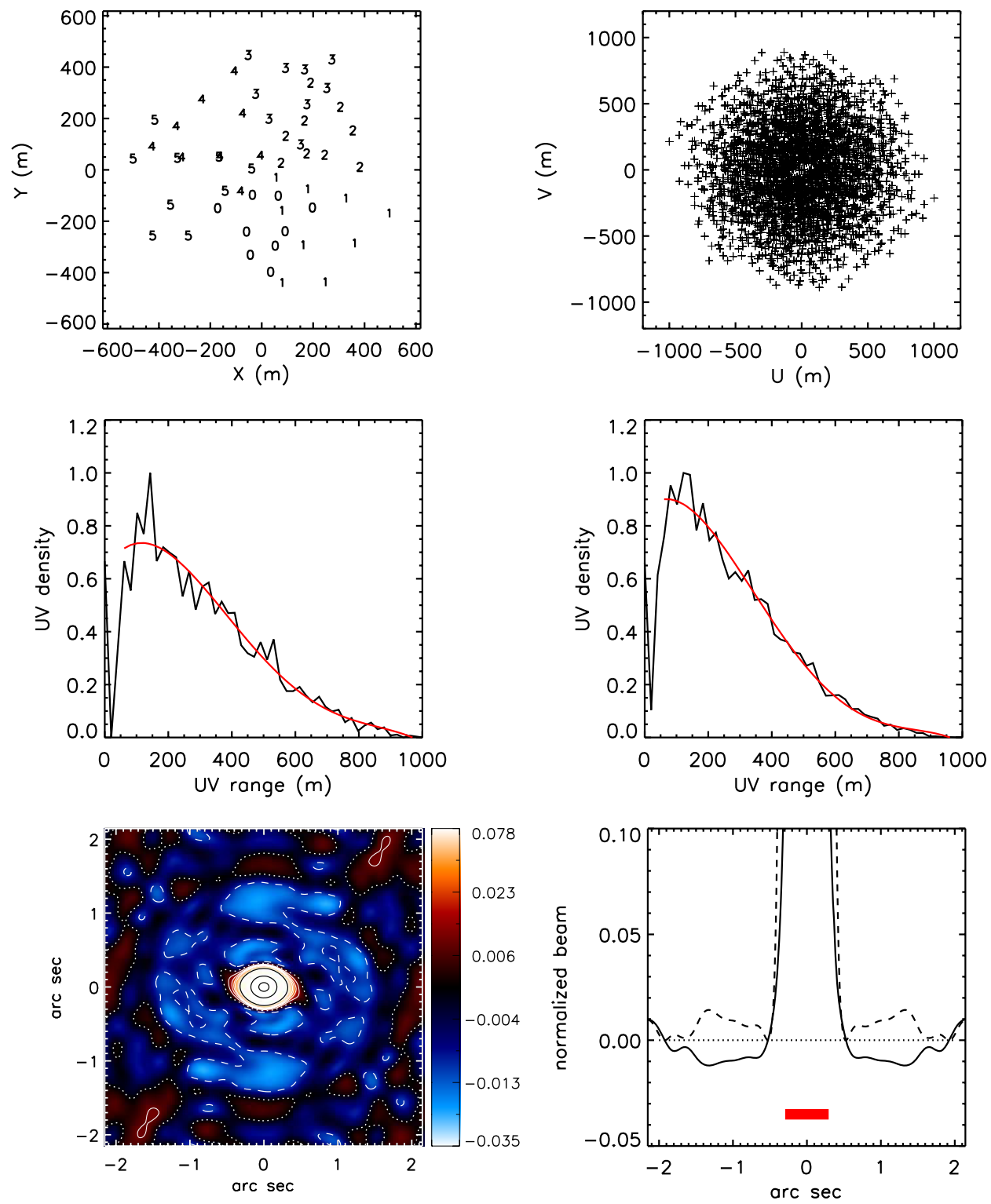

Fig. 9. Top left: Antenna locations, UV distribution, and beam for a 54-antenna s3p3d6 designed for low side lobes. Same format as Fig. 7. The beam power (dashed line) in the lower right panel has been multiplied by 10 . The figures of merit are listed in Table 4.

or Reuleaux triangle. Keto (1997) used a numerical algorithm to optimize the perturbations. Arendt et al. (2000) simply used random perturbations. Their slightly randomized CW-arrays look to be at least nearly as good as those designed by optimization, but a direct comparison has not been done.

In contrast, fully random patterns, rather than randomly perturbed patterns, generally do not have as good performance as more thoughtfully designed patterns. The reason is that random patterns may by chance position antennas too close to one another resulting in a concentration of separations and correspondingly higher side lobes as well as larger gaps elsewhere in the UV distribution.

However, it is easy to create arrays with random patterns, and there is an attractiveness to the savage simplicity of the process. For example, a Gaussian distribution of antenna locations produces a Gaussian distribution of separations because the auto-correlation function of two Gaussians is another Gaussian. This in turn provides a Gaussian beam through the Fourier transform relationship. A Monte Carlo algorithm can generate a large number of possible arrays from which the best can 

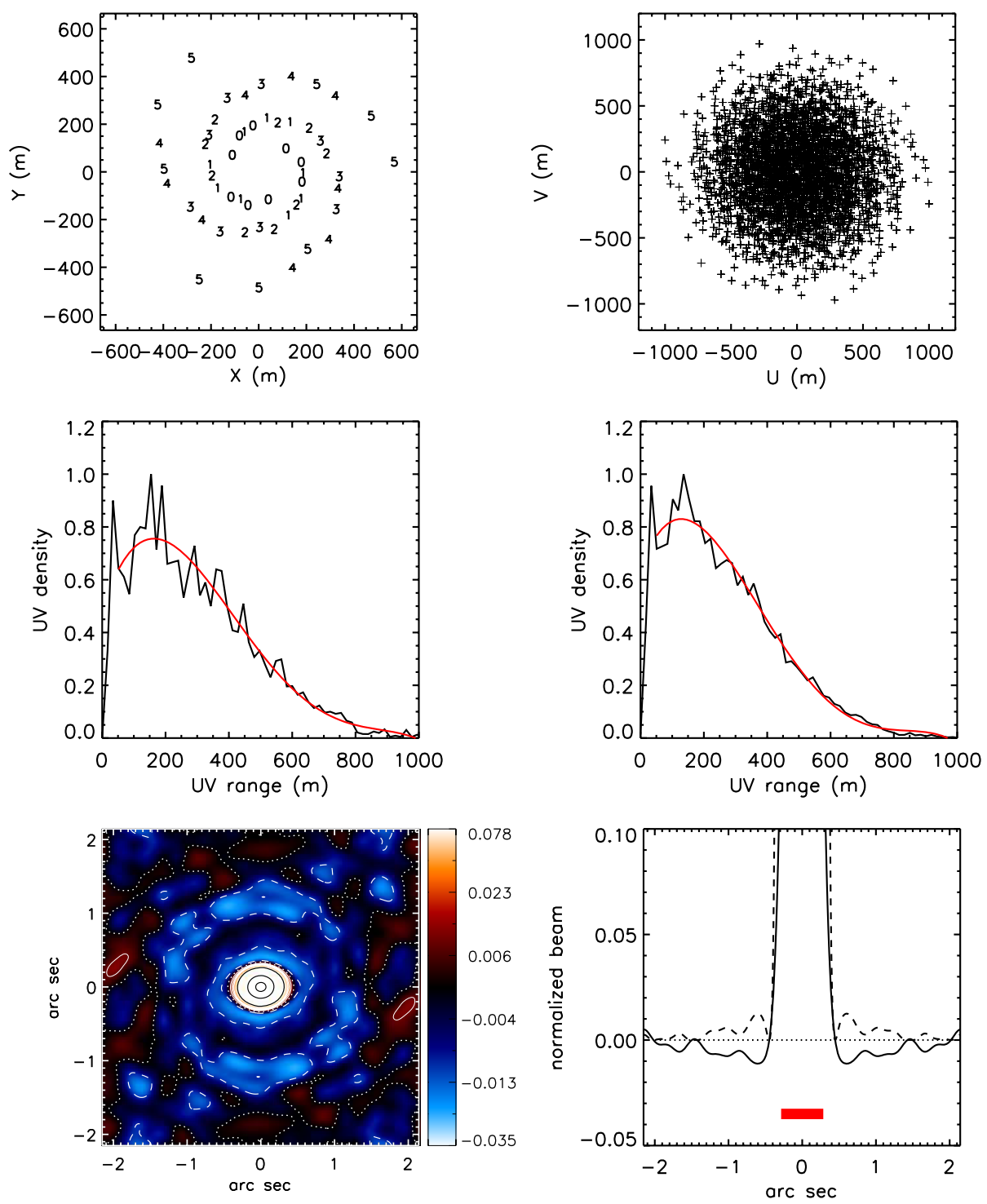

Fig. 10. Antenna locations, UV distribution, and beam for a 54-antenna spiral array designed for a high concentration of power in the main beam. The pattern uses the 9-element CW-array in Fig. 4 as the simple subarray. A power law scaling by a factor of 1.25 and a constant rotation of $164^{\circ}$ between subarrays produces the 3-armed pattern resembling a spiral galaxy. The difference between this array and the one in Fig. 11 is the amount of rotation between the scaled subarrays. Same format as Fig. 7. The beam power (dashed line) in the lower right panel has been multiplied by 100. The figures of merit are listed in Table 4.

be selected according to whatever criteria. Figure 14 shows an example selected for the best $K$-product from a very small number of trials, specifically 100 trials.

In contrast, a uniform distribution of antenna locations results in an array at the other end of our trade-off between high resolution and a concentrated beam. In one dimension, the autocorrelation function of a uniform distribution is a triangular function whose Fourier transform is the sinc function squared. The power pattern of the beam then follows the fourth power of the sinc. The beam of a 2-dimensional uniform distribution with a circular boundary is conceptually the same, approximately a $\operatorname{sinc}^{2}$ as a function of radius. An example of a two-dimensional array based on a uniform random distribution of antennas is shown in Fig. 14. This array had the best angular resolution out of 100 trials. The figures of merit are listed in Table 4 .

\section{Outriggers}

Interferometers are sometimes built with a few outrigger antennas around a main array to obtain 

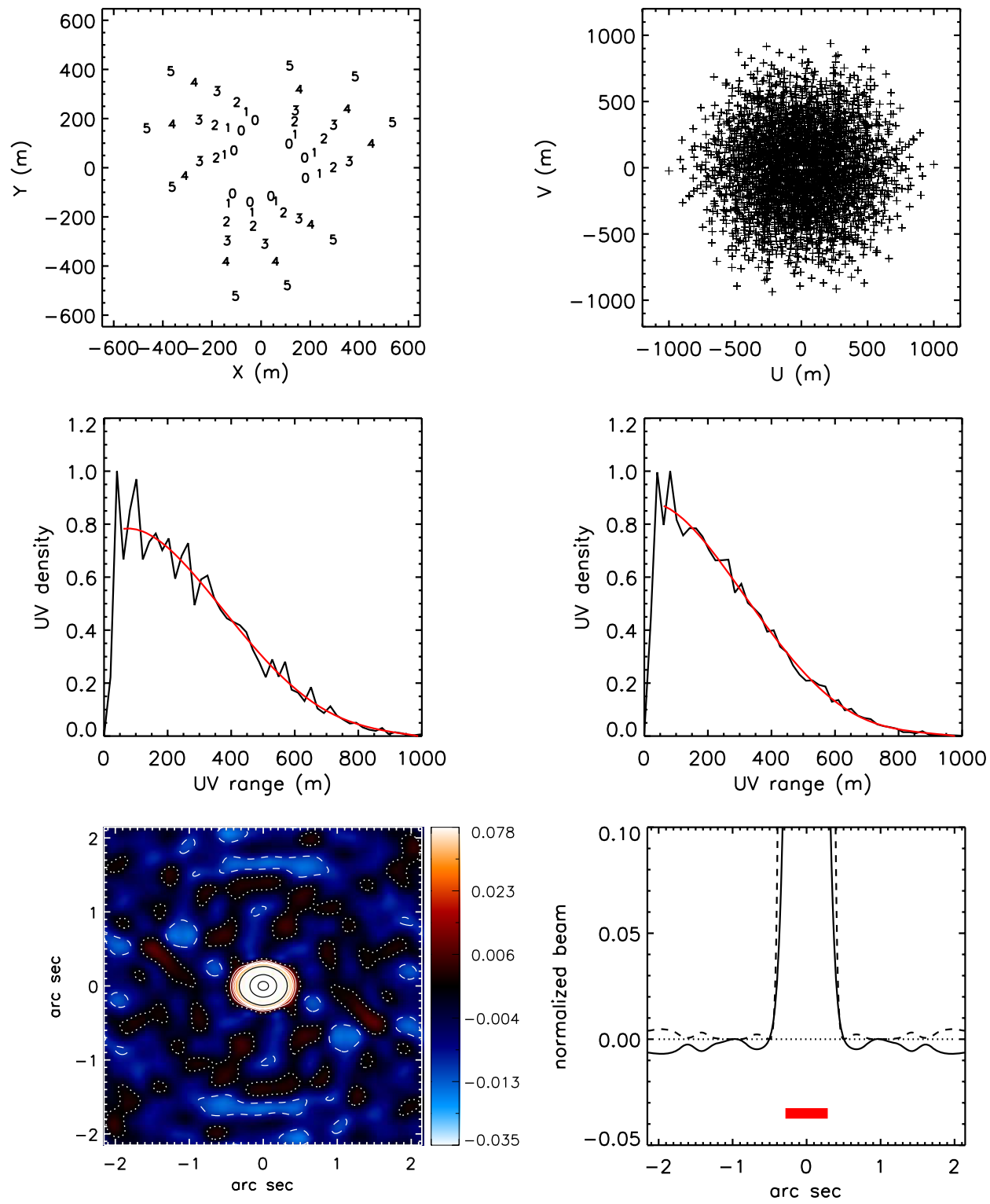

Fig. 11. Antenna locations, UV distribution, and beam for a 54-antenna spiral array designed for a high concentration of power in the main beam. The pattern uses the 9-element CW-array in Fig. 4 as the simple subarray. A power law scaling by a factor of 1.25 and a constant rotation of $113^{\circ}$ between subarrays produces the 9-armed pattern resembling sea stars. The difference between this array and the one in Fig. 10 is the amount of rotation between the scaled subarrays. Same format as Fig. 7. The beam power (dashed line) in the lower right panel has been multiplied by 100. The figures of merit are listed in Table 5 .

a limited sample of longer Fourier components. What do the H-arrays say about the placement of outriggers? H-arrays may be designed with concentric or asymmetric outriggers. Each has different properties.

Figure 15 shows a concentric configuration with the main array at the center of 6 outriggers. In this example, the main array is the 36 -antenna s6p6 array designed for high angular resolution; the outriggers use the locations of the 6-antenna subarray to make a 42 -antenna s6p $6+\mathrm{d} 6$ array. Figure 15 shows four concentric zones of UV coverage. First, on the largest scale, the 30 separations between the outriggers uniformly cover the UV space within the boundary. The next zone includes the separations between the outriggers and the main array, also colored red in Fig. 15. The two inner zones, blue and green in Fig. 15 (top right), are the separations of the main s6p6 H-array as described in Sec. 7.

In most applications, the inner UV points from the main array would be used in imaging and the outer UV points from the outriggers would be used 

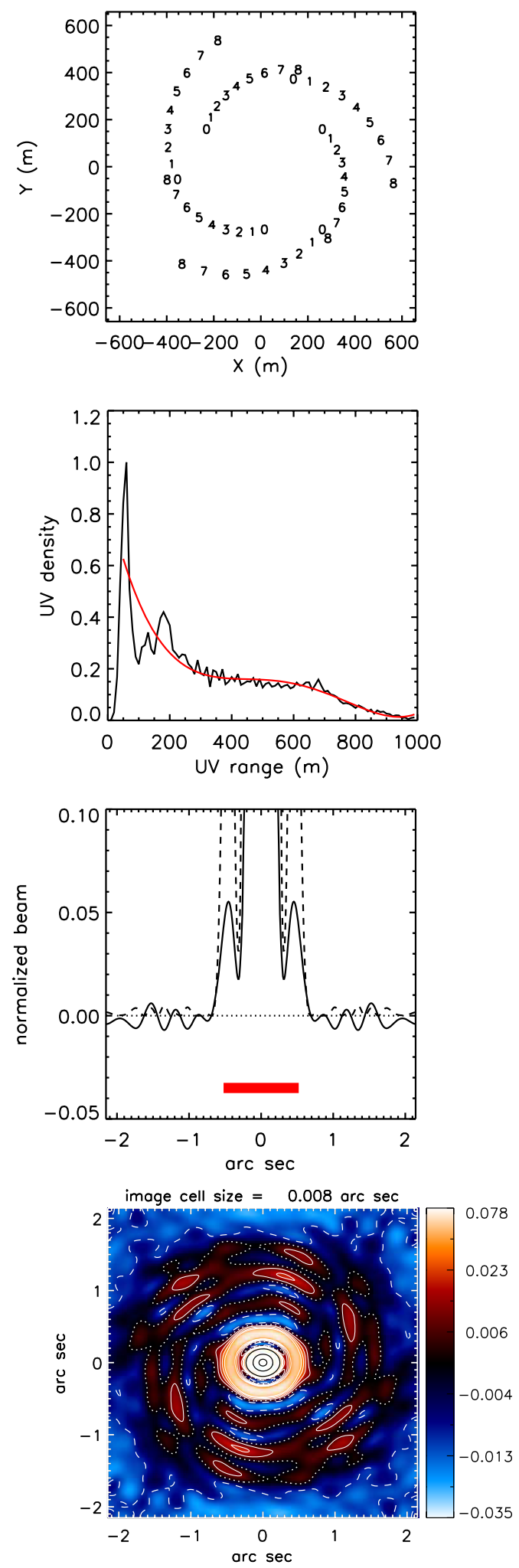
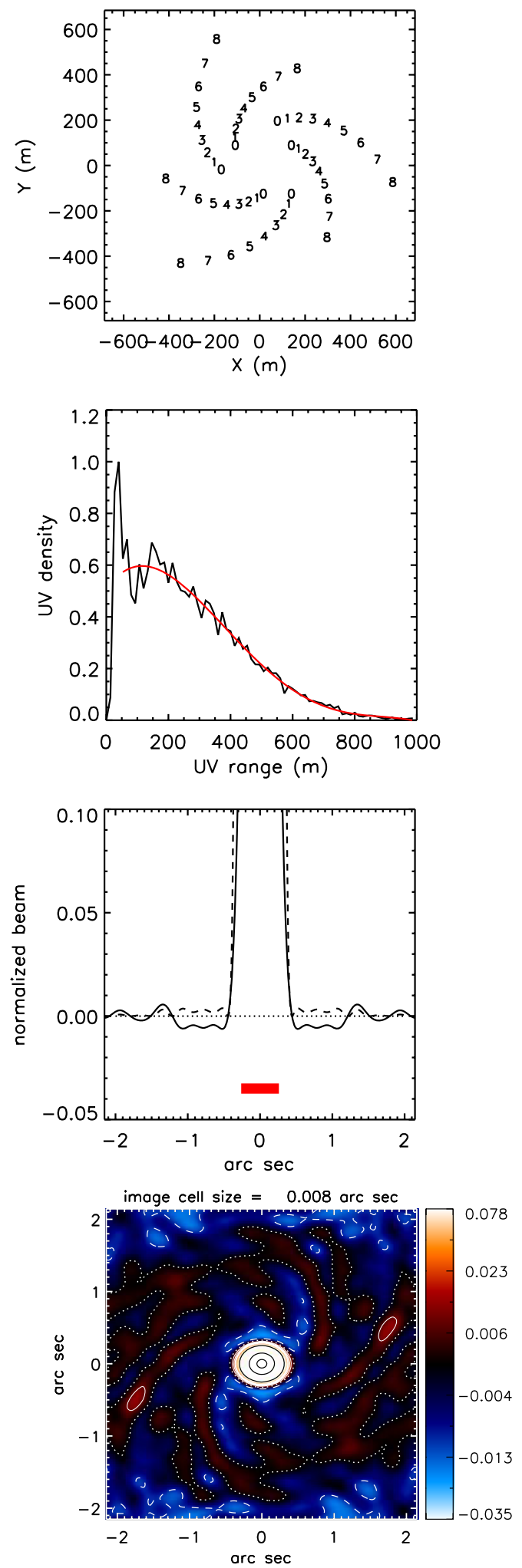

Fig. 12. Antenna locations, UV distribution, and beam for 54-antenna spiral arrays showing the effect of the power law scaling between subarrays. The pattern uses the 6-element CW-array in Fig. 3 as the simple subarray. In the left column the configuration is built by scaling successive subarrays by a factor of 1.05 . The right column shows the results for a scaling of by 1.15. The same arrays but with power law scalings of 1.25 and 1.35 are shown in Fig. 13. The lower scalings here produce beams of higher angular resolution. The panels have the same format as Fig. 7, except that here the UV coverage is not shown and the UV density is shown for ERS only. The beam power (dashed line) in the third panel down in both columns has been multiplied by 100 . The figures of merit are listed in Table 5 . 

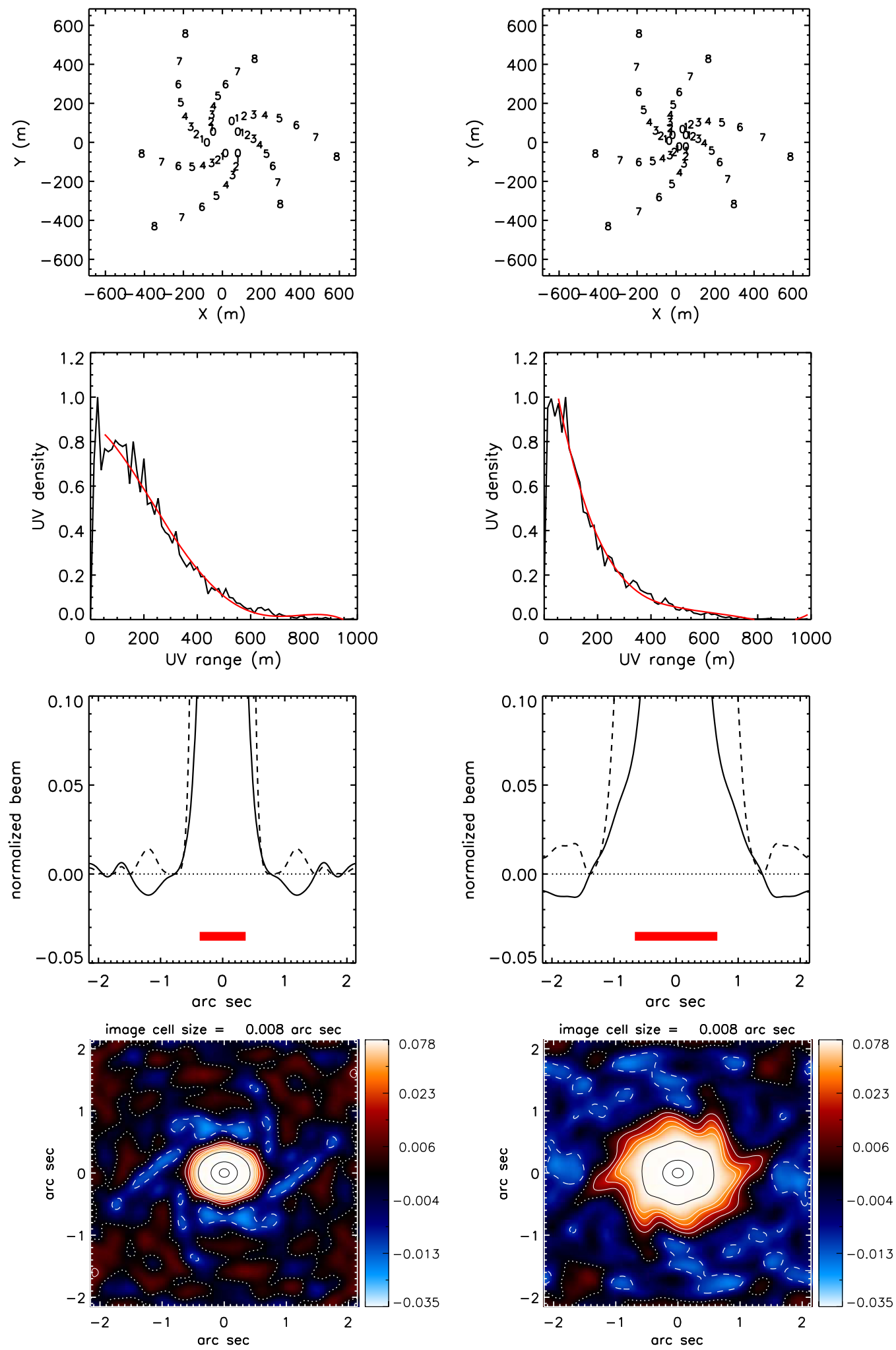

Fig. 13. Antenna locations, UV distribution, and beam for 54-antenna spiral arrays showing the effect of the power law scaling between subarrays. The pattern uses the 6-element CW-array in Fig. 3 as the simple subarray. In the left column the successive subarrays are scaled by a factor of 1.25 , and the right by 1.35 . The same arrays but with power law scalings of 1.05 and 1.15 are shown in Fig. 12. The higher scalings here produce beams of lower angular resolution. Same format as Fig. 12 . The beam power (dashed line) in the third panel down in both columns has been multiplied by 100. The figures of merit are listed in Table 4. 

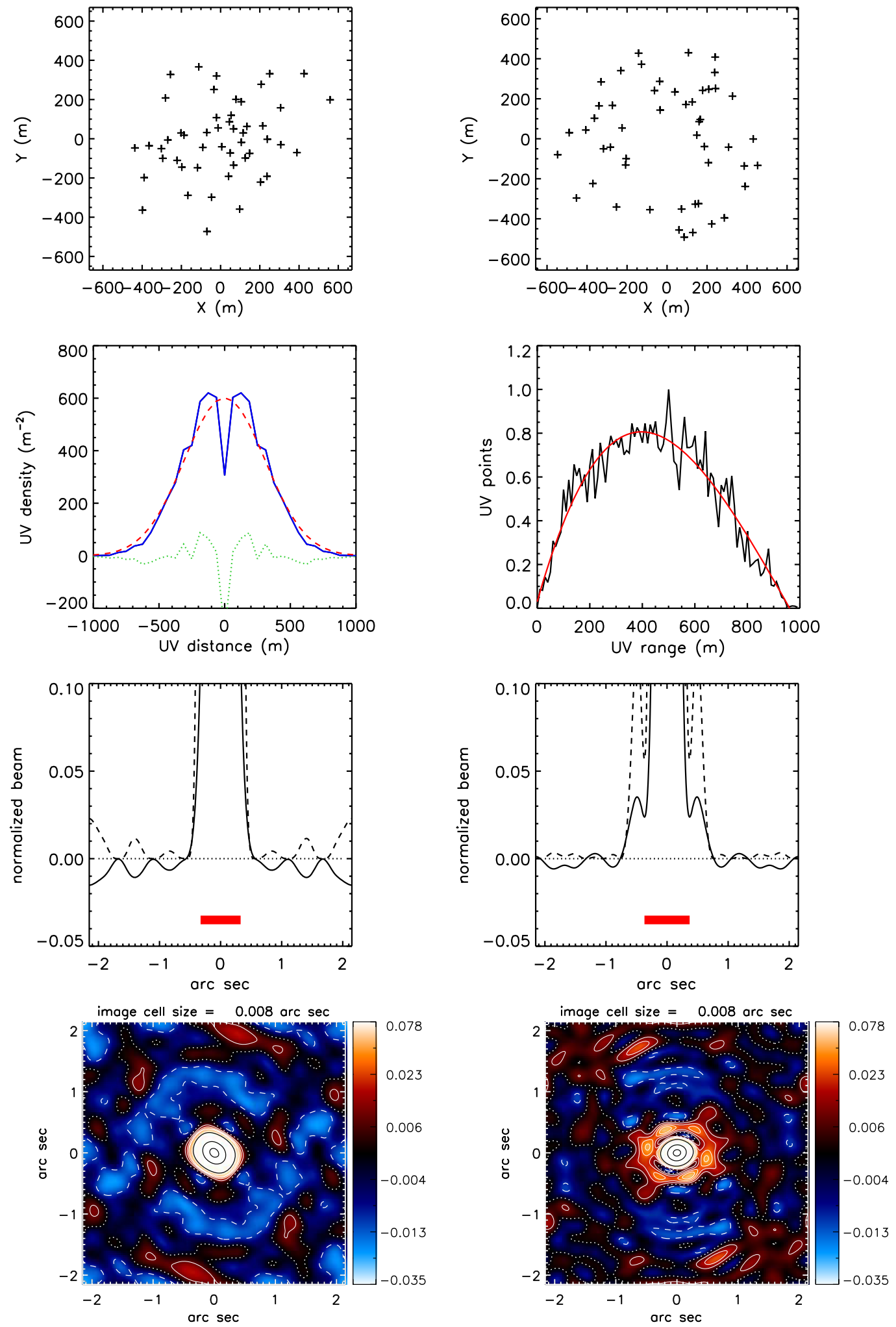

Fig. 14. (Color online) Antenna locations, UV distributions, and beams for two 54-antenna arrays with different random antenna locations. The left column shows an array created from a Gaussian or normal distribution of antenna locations. The right column shows an array with a uniform distribution of antennas. Same format as Fig. 7 except that the second panel in the left column plots a trace across the UV distribution (solid blue) to show the Gaussian distribution of antenna separations resulting from the Gaussian distribution of locations. The dashed red line shows a Gaussian fit and the dotted green line the residuals. The figures of merit are listed in Table 4. 

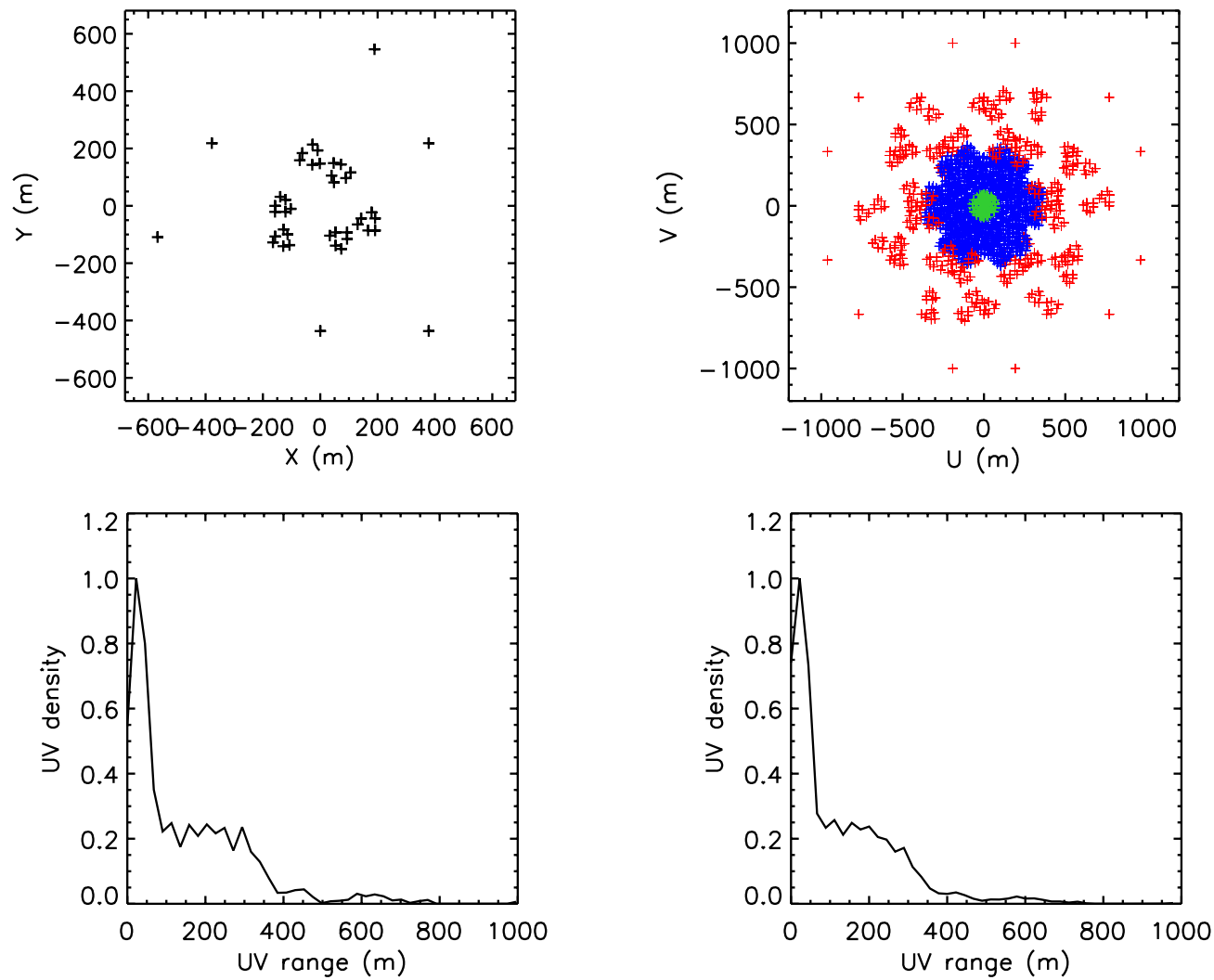

Fig. 15. (Color online) An s6p6-array with 6 concentric outriggers. The s6p6-array is the same as shown in Fig. 6, and the 6 outriggers take the positions of the 6-element subarray in Fig. 3 scaled by a factor of 5.5 times the first s-level subarray. Same format as Fig. 6 except the plots of the beam are not shown.

separately to locate point sources to high angular resolution. The outer UV points would generally not be used for imaging because they create uncanceled sine waves that do not improve the image fidelity. If the inner array, not including the outriggers, is scaled to $1000 \mathrm{~m}$ and the outriggers are not included in the Fourier transform of the beam, then the array design is the same as shown in Fig. 6, and the beam pattern and figures of merit are also the same. The UV coverage of the outriggers alone would be as shown in Fig. 3.

Alternatively, we can design an asymmetric outrigger array with the main array placed at one of the 6 locations of the 6 -element pattern as shown in Fig. 16. This creates a 41 antenna array, one less than the concentric design. This array again shows the four zones of UV coverage but has higher angular resolution, FWHM $=0.30$ arc seconds because the location of the main array on the border of the 6-element $\mathrm{CW}$ pattern creates longer spacings than the concentric placement. However, the UV coverage and the beam are asymmetric with more of the longer spacings in the north-south direction.
The asymmetric outrigger array results in better signal to noise on some of the longer separations whereas the concentric design improves some of the shorter.

\section{Beam Patterns with Different Observations}

How does the performance of an array change for different types of observations, for example when tracking targets at different declinations that do not pass directly overhead? In astronomical imaging, the beams of good arrays, those that have a good distribution of antenna separations, generally maintain their good properties when observing sources in either instantaneous (snapshot) imaging or Earth rotation synthesis and also when observing at different astronomical declinations.

The previous examples show the beam patterns for a source that transits through zenith. Figure 17 shows the beam patterns for two low side lobe designs, the 36 -antenna s6p6, and the 54antenna s3p3d6 configurations, tracking sources at 

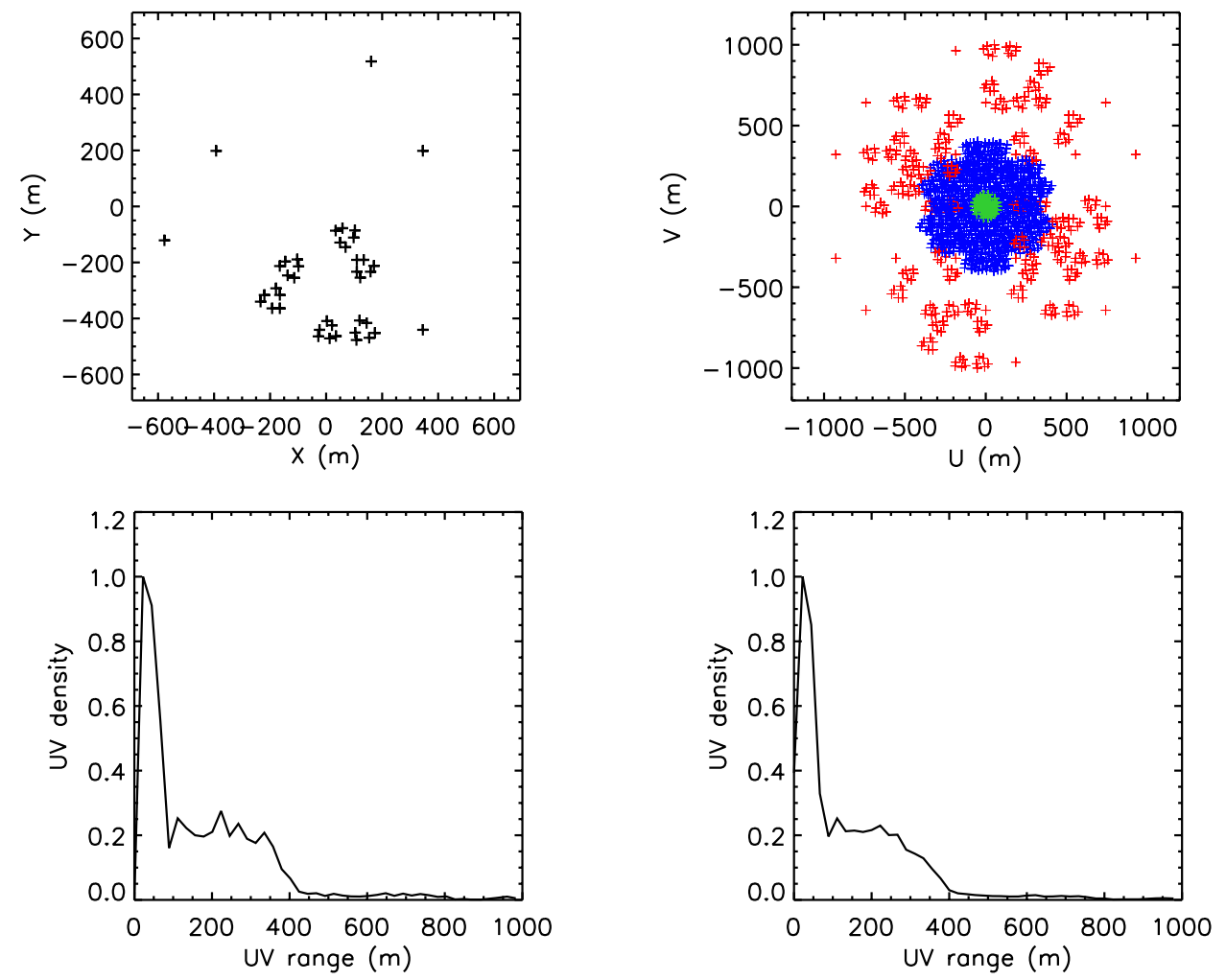

Fig. 16. An s6p6-array with 5 asymmetric outriggers. The s6p6-array is the same as shown in Fig. 6, and the 5 outriggers take 5 of the positions of the 6-element subarray in Fig. 3 scaled by a factor of 5.5 times the first s-level subarray. The main s6p6-array takes the sixth position of the s-level subarray. Same format as Fig. 15.
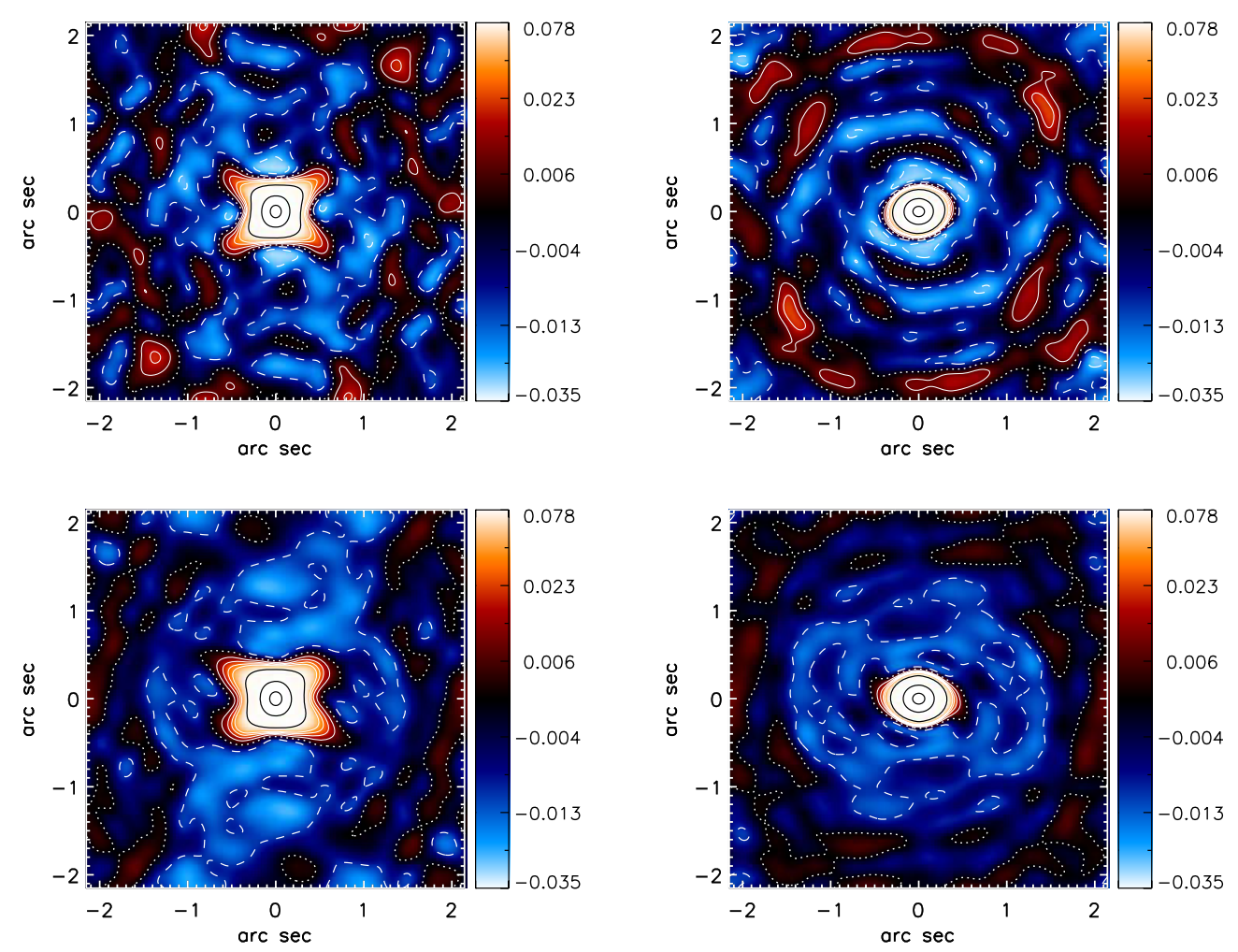

Fig. 17. Beam patterns for $3 \mathrm{H}$-arrays tracking targets to the north (right) and south (left). Top and bottom show the beam patterns for the 36 antenna configuration s6p6 of Fig. 7 and the 54 antenna configuration s3p3d6 of Fig. 9, respectively. 
declinations of $-23^{\circ}$ and $+46^{\circ}$ assuming that the array is at a latitude of $+23^{\circ}$. The FWHM changes with declination as the baselines are shortened by projection, but the arrays generally maintain their good characteristics.

\section{Numerical Optimization}

These examples show how easy it is to build $\mathrm{H}$-arrays with excellent performance without numerical optimization, but the arrays could still be improved. The optimization problem is much simpler with $\mathrm{H}$-arrays because the number of parameters that describe the arrays, the relative scaling of the levels and the subarrays, and the rotation of the subarrays, is fewer than the number of antennas. For example, the description of a threelevel array with 216 antennas requires 14 numbers: two scalings between the levels, and six scalings and six rotations for the subarrays. An H-spiral can be described by as few two numbers. In contrast, the description of the antenna locations individually requires 432 numbers for the $216 \mathrm{X}$. Y pairs. Optimizing the antenna positions with respect to each other is a combinatorially explosive problem with the number of possible configurations increasing exponentially with the number of antennas. It is difficult for algorithms that optimize the antenna positions to demonstrate convergence to a global minimum. One difficulty is that quasi-random configurations with quasi-Gaussian distributions represent particularly seductive local minima. Another problem is that ordered patterns such as the Reuleaux triangle require a degree of coherence that is difficult to obtain for algorithms that move one antenna at a time.

\section{Comparison to Other Designs}

It is worth comparing these example $\mathrm{H}$-arrays to other designs. The recently constructed Atacama Large Millimeter Array (ALMA) uses a variety of different configurations listed in their CASA simulation software. Three of them, configurations ALMA-02, ALMA-14, and ALMA-28, are shown in Figs. 18-20. Aside from their different diameters, here normalized to $1000 \mathrm{~m}$, these three configurations are quite different from one another reflecting their different design goals as mentioned in Sec. 3. Configuration ALMA-02 is designed to
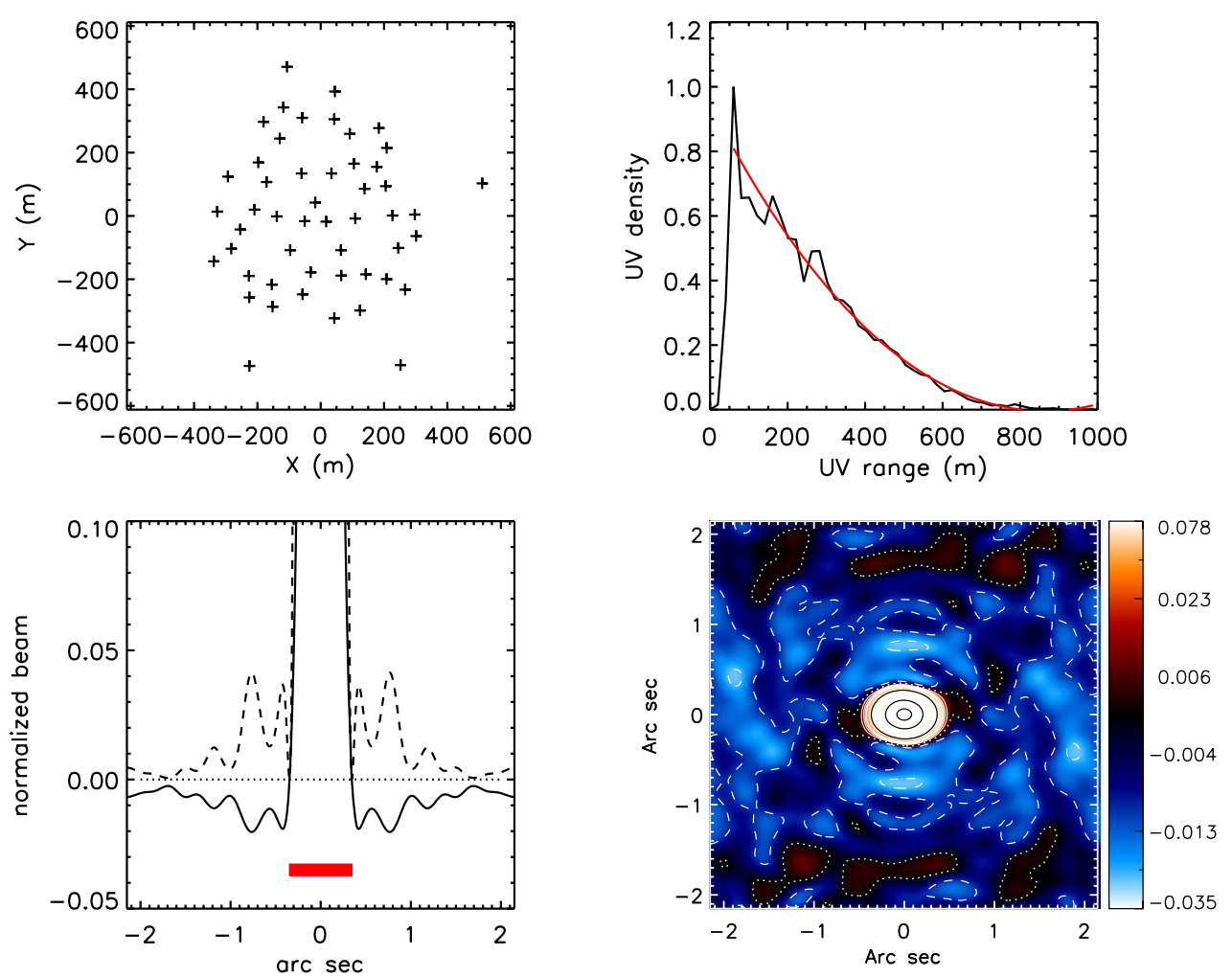

Fig. 18. Top left: Antenna locations, UV-distribution, encircled energy, and beam pattern for the ALMA-02 configuration. Same format as Fig. 12. The figures of merit are listed in Table 4. 

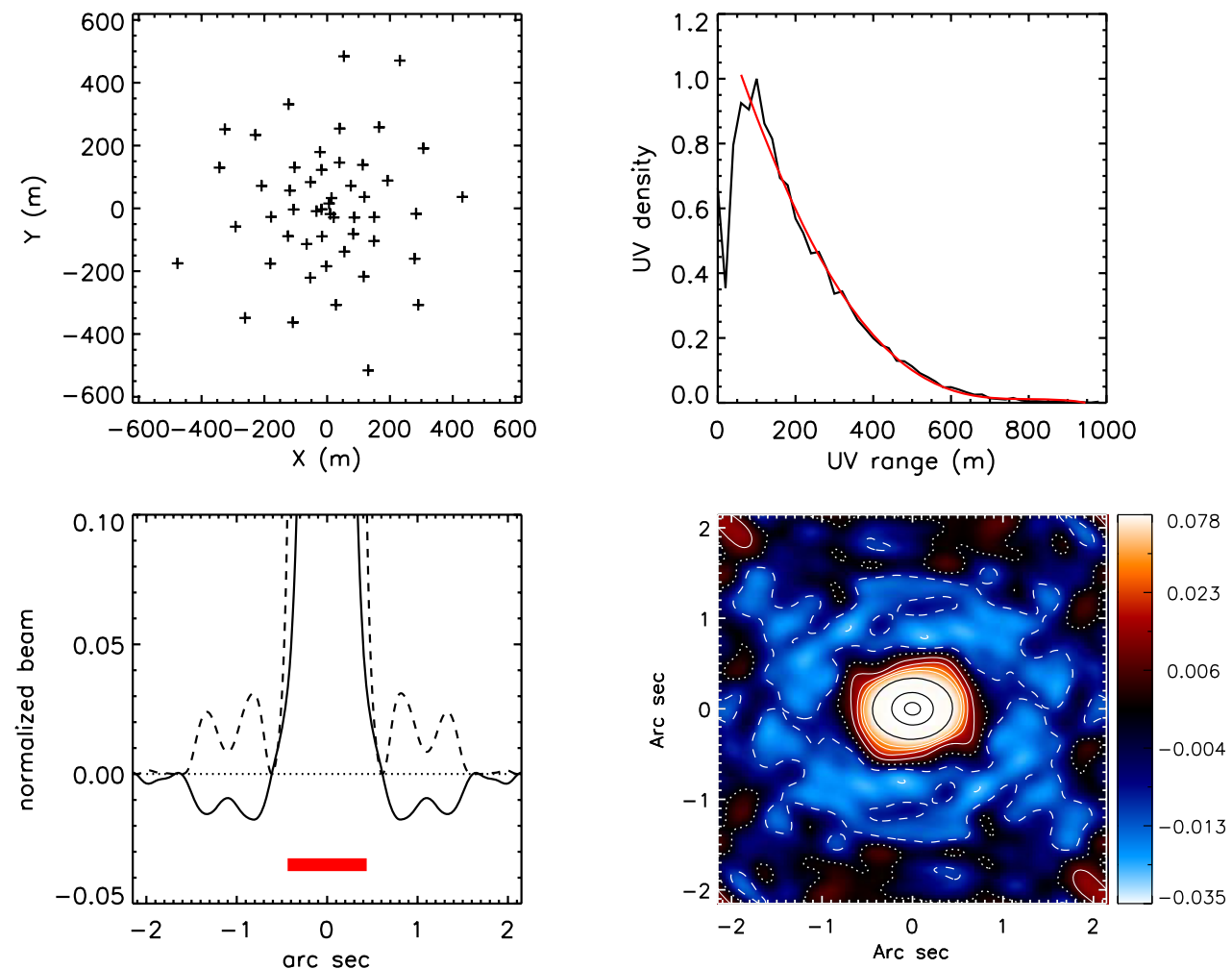

Fig. 19. Top left: Antenna locations, UV-distribution, encircled energy, and beam pattern for the ALMA-14 configuration. Same format as Fig. 12. The figures of merit are listed in Table 4.
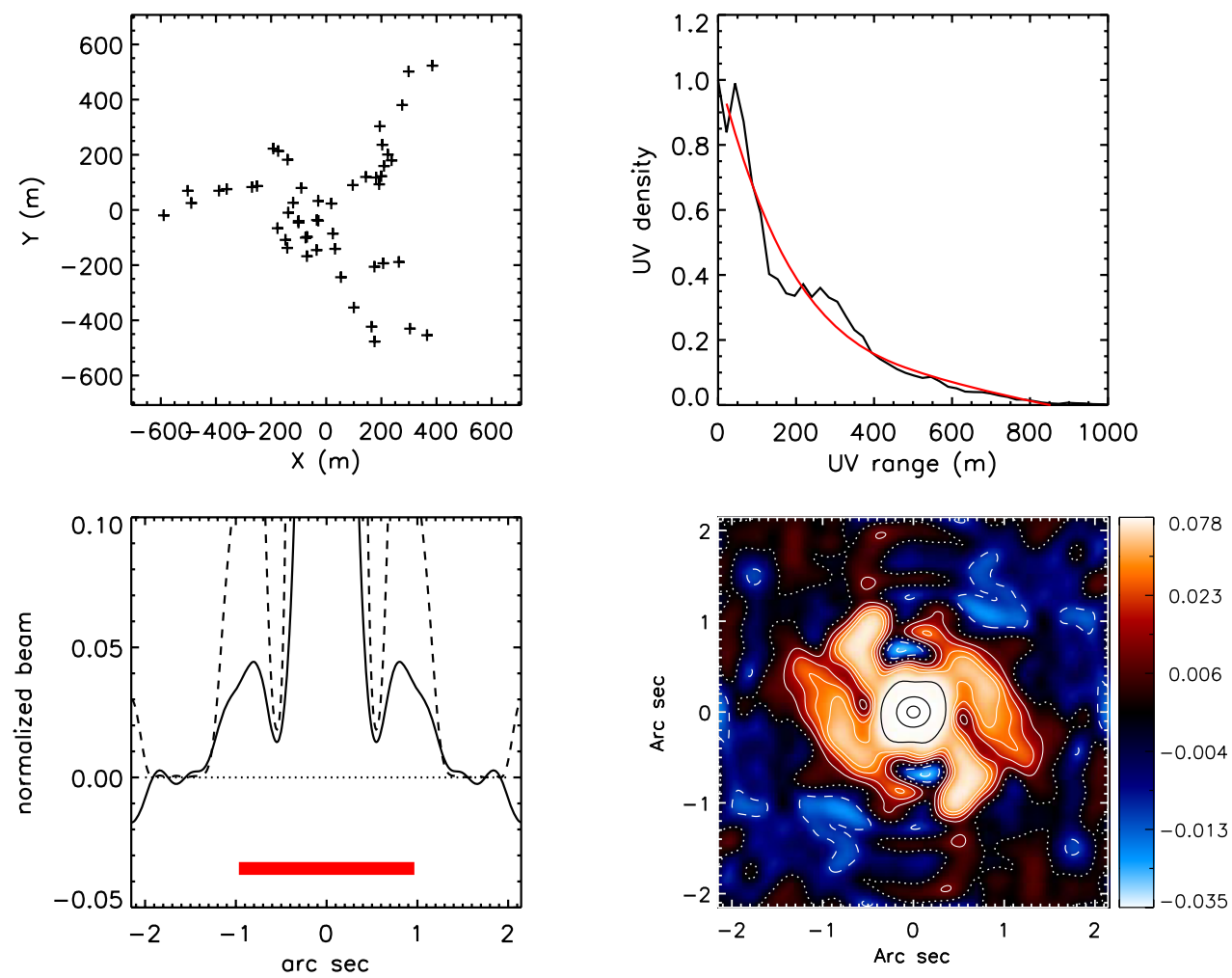

Fig. 20. Top left: Antenna locations, UV-distribution, encircled energy, and beam pattern for the ALMA-28 configuration. Same format as Fig. 12. The figures of merit are listed in Table 4. 
Table 4. Figures of merit for example arrays in Earth rotation synthesis.

\begin{tabular}{|c|c|c|c|c|c|c|}
\hline Array name & $\begin{array}{c}\text { Number of } \\
\text { antennas }\end{array}$ & $\begin{array}{l}\text { Shown as } \\
\text { figure }\end{array}$ & $\begin{array}{l}\text { FWHM } \\
(\operatorname{arc~sec})\end{array}$ & $\begin{array}{c}\text { Radius of } 98 \% \\
\text { encircled energy } \\
\text { (arc sec) }\end{array}$ & $\begin{array}{c}K_{98} \text { product } \\
(\mathrm{m} \text { arc sec })\end{array}$ & $\begin{array}{l}\text { Normalized } \chi^{2} \\
\quad(\times 1000)\end{array}$ \\
\hline$J_{1}(r) / r$ & $\ldots$ & 1 left & 0.27 & 1.53 & 1530 & $\ldots$ \\
\hline Gauss & $\ldots$ & 1 right & 0.40 & 0.35 & 353 & $\ldots$ \\
\hline s6p6 & 36 & 6 & 0.17 & 1.38 & 1379 & 1.70 \\
\hline s6p6 & 36 & 7 & 0.21 & 0.29 & 285 & 41.5 \\
\hline s3p3d6 & 54 & 8 & 0.17 & 0.89 & 891 & 0.20 \\
\hline s3p3d6 & 54 & 9 & 0.23 & 0.29 & 294 & 1.79 \\
\hline H-spiral galaxy & 54 & 10 & 0.23 & 0.29 & 285 & 1.47 \\
\hline H-spiral sea star & 54 & 11 & 0.23 & 0.29 & 285 & 0.81 \\
\hline Gauss random & 50 & 14 & 0.26 & 0.33 & 327 & 7.80 \\
\hline unif. random & 50 & 14 & 0.20 & 0.37 & 369 & 6.11 \\
\hline ALMA-02 & 50 & 18 & 0.26 & 0.35 & 353 & 1.74 \\
\hline ALMA-14 & 50 & 19 & 0.29 & 0.44 & 437 & 0.79 \\
\hline ALMA-28 & 50 & 20 & 0.25 & 0.97 & 967 & 2.36 \\
\hline
\end{tabular}

Table 5. Figures of merit for spirals based on a 6-element subarray with different scaling.

\begin{tabular}{ccccccc}
\hline $\begin{array}{c}\text { Scaling } \\
\text { factor }\end{array}$ & $\begin{array}{c}\text { Number of } \\
\text { antennas }\end{array}$ & $\begin{array}{c}\text { Shown as } \\
\text { figure }\end{array}$ & $\begin{array}{c}\text { FWHM } \\
(\operatorname{arc~sec})\end{array}$ & $\begin{array}{c}\text { Radius of 98\% } \\
\text { encircled energy } \\
(\operatorname{arc~sec})\end{array}$ & $\begin{array}{c}K_{98} \text { product } \\
(\mathrm{m} \text { arc sec })\end{array}$ & $\begin{array}{c}\text { Normalized } \chi^{2} \\
(\times 1000)\end{array}$ \\
\hline 1.05 & 54 & 12 left & 0.18 & 0.52 & 521 & 5.20 \\
1.15 & 54 & 12 right & 0.23 & 0.26 & 370 & 0.88 \\
1.25 & 54 & 13 left & 0.27 & 0.37 & 664 & 0.84 \\
1.35 & 54 & 13 right & 0.32 & 0.66 & 0.43 \\
\hline
\end{tabular}

minimize side lobe levels. ALMA-14 is a spiral modified to approximate a Gaussian beam. ALMA-28 is a Y-pattern similar to the VLA. Both ALMA-02 and ALMA-14 have side lobe levels comparable to the 36 or 54 antenna $\mathrm{H}$-arrays, but the $\mathrm{H}$-arrays, the appropriately scaled $\mathrm{H}$-spirals, as well as the Gaussian random array, all have better angular resolution and a tighter concentration of beam energy encircled in a smaller radius. The figures of merit for the three ALMA configurations are listed in Table 4.

\section{Conclusions}

(1) Different designs for cross-correlation interferometer arrays exist in a continuous space of trade-offs between competing goals of high angular resolution, concentrated beam power, compact array size, and the smoothness of the distribution of the measured Fourier components.

(2) Three figures of merit are useful in assessing the imaging performance of an array. (a) The $K_{n n}$ product of the array size and the radius which encompasses $n n$ of the total beam energy.

(b) The angular resolution as measured by the beam FWHM.

(c) The smoothness of the distribution of antenna separations.

(3) A technique of building a large array by scaling and rotating one simple pattern of a small number of antenna locations can be used to construct hierarchical arrays (H-array) or spiral arrays (H-spirals) with a large number of antennas.

(4) Hierarchical arrays (H-arrays) provide an excellent framework for locating the antennas of a cross-correlation imaging interferometer to provide optimal imaging qualities.

(5) By changing the scaling between the subarrays, both the $\mathrm{H}$-arrays and the $\mathrm{H}$-spirals can produce beams at the particular point along the trade-off between angular resolution and concentrated beam power. 
(6) Sparsely populated $\mathrm{H}$-arrays also have excellent performance and are useful for interferometers designed with more antenna locations than antennas. This allows the angular resolution to be changed by populating different subsets of the antenna locations.

(7) The construction procedure of scaling and rotating a basic subarray can also be used to design high performance, intelligent spiral arrays, H-spirals. Spirals are good for array designs emphasizing low side lobe designs rather than high angular resolution.

(8) The H-arrays are easy to optimize because they can be described by a few parameters, much fewer than the number of antennas themselves.

(9) H-arrays are useful for future multi-element interferometers.

\section{References}

Arendt, R. G., Fixsen, D. J., Moseley, S. H., 2000, Ap. J., 536, 500 .

Bracewell, R., 1958, Proc. of the IRE, 46, 97.
Bracewell, R., 1999, The Fourier Transform and Its Applications, McGraw-Hill.

Conway, J., 2006, ALMA Document 2006-10-17-ALMA90.02.00.00-006-A-SPE.

Guilloteau, S. et al., 1991, A\&A, 262, 624.

Helfer, T., 2004, CARMA Memorandum Series number 20, version 2 .

Hogbom, 1974, A\&A Suppl., 15, 417.

Holdaway, 2007, ALMA Document 2006-10-17-ALMA90.02.00.00-006-A-SPE.

Ishiguro, M., 1980, Radio Science, 15, 1163.

Keto, E., 1997, Ap. J., 475, 843.

Kogan, L., 2000, IEEE Trans. Antennas $\&$ Propagation, 48, 1075.

Mills, B. Y. \& Little, A. G., 1953, Aust. J. Phys., 6, 272.

Millenaar, R. P., Bolton, R. C. \& Lazio, J., 2011, SKA document number WP3-050.020.010-TR-001, Array Configurations for Candidate SKA Sites: Design and Analysis.

Moffett, A. T., 1968, IEEE Trans. Ant. Prop., 16, 172.

Padin, S. et al., 1991, PASP, 103, 461.

Ryle, M., 1962, Nature, 194, 517.

Ryle, M. \& Hewish, A., 1960, MNRAS, 120, 220.

Thompson, A. R., Clark, B. G., Wade, C. M. \& Napier, P. J., 1980, ApJS, 44, 151.

Welch, W. J. et al., 1996, PASP, 108, 93.

Wooten, A. \& Thompson, A. R., 2009, Proc. IEEE, 97, 1463. 